\title{
Effects of 1-Methylcyclopropene and Controlled Atmosphere on Ethylene Synthesis and Quality Attributes of Avocado cvs. Edranol and Fuerte
}

\author{
Daniela Olivares, ${ }^{1}$ Edgard Alvarez, ${ }^{1}$ Daniela Véliz, ${ }^{1}$ Miguel García-Rojas, ${ }^{1}$ Camila Díaz, \\ and Bruno G. Defilippi (iD) \\ ${ }^{1}$ Instituto de Investigaciones Agropecuarias INIA-La Platina, Santa Rosa, 11610 Santiago, Chile \\ ${ }^{2}$ Universidad Santo Tomás, Facultad de Agronomía, Ejército Libertador 146, Santiago, Chile \\ Correspondence should be addressed to Bruno G. Defilippi; bdefilip@inia.cl
}

Received 16 October 2019; Revised 22 December 2019; Accepted 13 January 2020; Published 24 February 2020

Academic Editor: Alejandro Hernández

Copyright (c) 2020 Daniela Olivares et al. This is an open access article distributed under the Creative Commons Attribution License, which permits unrestricted use, distribution, and reproduction in any medium, provided the original work is properly cited.

\begin{abstract}
Avocado production worldwide relies on several varieties, with "Hass" being the most commercialized; however, the available genotypes include a number of green-skin varieties with important roles in several countries. Because many technologies have already been developed in "Hass" avocado, the main objective of this study was to evaluate the effects of controlled atmosphere (CA) storage and 1-methylcyclopropene (1-MCP) application during long-term storage of "Edranol" and "Fuerte" avocados. Fruits of both varieties were harvested at two maturity stages: an early harvest close to $20-23 \%$ dry matter (DM) content and another after two months, with 22\% and 32\% DM content for Edranol and Fuerte, respectively. After harvest, the fruit was stored under the following conditions: (i) regular air storage (RA), (ii) CA with $4 \% \mathrm{O}_{2}$ and $6 \% \mathrm{CO}_{2}$, and (iii) 1-MCP applied at 300 ppm. Avocados were stored at $5{ }^{\circ} \mathrm{C}$ and $85 \%$ relative humidity. Physiological and quality evaluations were performed immediately after 30 and 50 days; afterwards, the avocados were maintained at $20^{\circ} \mathrm{C}$ (shelf life) until they reached the ready-to-eat stage. Ethylene synthesis was assessed by measuring the transcript accumulation of the ACO and ACS genes. The two varieties showed distinct respiration and ethylene production rates during ripening, and fruit stored under CA or after application of 1-MCP showed lower respiration rates than fruit stored under RA, with the lowest rate in 1-MCP-treated avocados. ACS and ACO transcript levels were also lower under both conditions. CA and 1-MCP were very effective tools for extending storage life mainly by reducing the fruit softening rate and the incidence of pulp disorders in both varieties, and interestingly, these techniques did not severely affect the days to reach the ready-to-eat stage. Therefore, the use of CA and 1-MCP technologies in "Fuerte" and "Edranol" seems to be suitable for maintaining quality through 50 days of storage.
\end{abstract}

\section{Introduction}

Avocado production worldwide relies on several varieties originating from the West Indian, Guatemalan, and Mexican races [1]. Among them, the most commercialized variety worldwide is "Hass," which is characterized, among other things, by the development of a black skin at the ripe stage. The economic importance of the "Hass" variety is also seen in the amount of research performed worldwide and in the development of technologies that focus on this variety. However, among the genotypes available, there are a significant number of green-skin varieties that have important roles in several countries, including European and South American countries, where consumers prefer these "Hass" avocados [2]. Additionally, green-skin varieties are the important varieties when "Hass" is not available in the market.

Both black- and green-skin varieties need to maintain quality attributes, including appearance (size, color, and absence of damage), texture (mainly firmness), internal quality (color, absence of physiological disorders, and decay), and flavor after harvest. Among these quality attributes, 
the changes during ripening are modulated by ethylene, with pulp softening and skin color being the most studied due to their importance in limiting storage potential and commercialization. Additionally, changes in quality attributes are affected by growing conditions during fruit development on the tree, i.e., cultural management and climatic conditions, and especially by factors during harvest and storage, such as maturity stage, temperature, relative humidity, length of storage, or postharvest technologies used during this period, among others [3].

Among the technologies available for extending the storage potential of avocado, most rely on the capacity to reduce fruit metabolism by affecting the respiration rate and ethylene production and perception. The most important technology available is the use of low temperature, and this method is the basis for postharvest management of avocado, which is highly studied worldwide. However, the extended storage life achieved with low-temperature treatment alone is not sufficient for reaching distant markets as it results in excessive pulp softening, color change, and the development of internal damage due to early ripening, senescence, and decay. Therefore, at least for avocado, there is a need to use additional postharvest technologies, such as a controlled atmosphere (CA) and ethylene inhibitors, such as 1-methylcyclopropene (1-MCP).

CA treatment is widely used on "Hass" avocado, and it is based on reducing oxygen levels and increasing carbon dioxide during storage or transit [4]. These changes affect ethylene synthesis by reducing the enzyme activity of 1-aminocylpropane carboxylic acid oxidase (ACO), which catalyzes a key step in ethylene production. Depending on the levels of both gases, there are several benefits, such as a reduction in the softening rate and physiological disorders and a delay in color development and the prevalence of decay. For example, in "Edranol" and "Fuerte," Bower et al. [5] showed that a concentration of $6 \% \mathrm{O}_{2}$ and $4 \% \mathrm{CO}_{2}$ or $2 \% \mathrm{O}_{2}$ and $10 \% \mathrm{CO}_{2}$ extended postharvest life by $7 \mathrm{~d}$ compared with storage under RA $[6,7]$. The effectiveness of CA treatment is also influenced by the quality of the raw material at harvest, the maturity stage, and the length and temperature of storage, among other factors.

1-MCP, an ethylene perception antagonist, has been extensively used in multiple species to extend storage life due to its remarkable effects on the active sites of ethylene perception, altering ethylene-dependent processes such as softening and color development, among others $[8,9]$. In avocado, 1-MCP has shown positive effects in delaying not only pulp softening and color development but also the development of physiological disorders such as internal browning [10-12]. However, in some varieties, such as "Hass," "Pinkerton," and "Ryan," 1-MCP has been reported to exert a negative effect by extending the time in which the fruit reaches the ready-to-eat stage, resulting in excessive water loss, uneven ripening, and decay $[6,8,13]$. Therefore, presently, 1-MCP is used commercially in only some countries, such as South Africa, or with several restrictions in other avocado growing countries.

Because most of the above-mentioned technologies have been evaluated previously in "Hass" avocado, the main objective of this study was to evaluate the effects of CA and 1-MCP during long-term storage of "Edranol" and "Fuerte" avocados harvested at two maturity stages.

\section{Materials and Methods}

2.1. Materials. "Fuerte" and "Edranol" avocados were harvested from a commercial orchard located in Ocoa $\left(32^{\circ} 50^{\prime} 16^{\prime \prime} \mathrm{S} 71^{\circ} 2^{\prime} 21^{\prime \prime} \mathrm{W}\right)$, Chile. During fruit growth, dry matter (DM) content was measured monthly to assess the maturity stage of both varieties (Figure 1). This parameter was used as the harvest index and was determined by drying the samples in an oven at $103^{\circ} \mathrm{C}$ for 24 hours until a constant weight was reached [14]. Fruits of both varieties were harvested at two maturity stages based on DM content: an early harvest close to a DM of 20-23\% (Harvest I) and another after two months (Figure 1) with higher levels (Harvest II). The DM content and fruit characteristics for both varieties at harvest are shown in Figure and Table 1.

After harvest, the fruit was transported at $20^{\circ} \mathrm{C}$ within a few hours to the Postharvest Laboratory facilities at the Institute of Agricultural Research (INIA). Fruits were randomly selected and subjected to the following postharvest treatments: (i) regular air (RA) storage, (ii) controlled atmosphere (CA) storage with $4 \% \mathrm{O}_{2}$ and $6 \% \mathrm{CO}_{2}$ using a static automatic gas control unit, and (iii) 1-MCP application at harvest and storage under RA. The avocados were packaged in ventilated plastic boxes and stored at $5^{\circ} \mathrm{C}$. The relative humidity $(\mathrm{RH})$ values for RA and CA were $80-85 \%$ and higher than $90 \%$, respectively. Evaluations were performed after 30 and $50 \mathrm{~d}$, and after each storage time, fruit samples were maintained at $20^{\circ} \mathrm{C}$ and $45 \% \mathrm{RH}$ (shelf life) until reaching the ready-to-eat stage (firmness less than or equal to $9.8 \mathrm{~N}$ ) [3]. For 1-MCP application at harvest, 1-MCP (SmartFresh $^{\mathrm{TM}}$ and Agrofresh) was applied in a sealed container at $300 \mathrm{ppb}$. 1-MCP was applied for 12 hours at $5^{\circ} \mathrm{C}$ [8]. Three replicates per treatment were used, with one replicate corresponding to 10 or 35 avocados according to the variable evaluated.

\subsection{Determination of Ethylene Production and Respiration} Rates. At each evaluation time, the respiration and ethylene production rates were measured individually in 10 fruits per treatment. After removal from cold storage, fruit were exposed for 2 hours in a room at $20^{\circ} \mathrm{C}$ until they reached a flesh temperature close to $20^{\circ} \mathrm{C}$. To determine the ethylene production rate, each avocado was placed in a $1.6 \mathrm{~L}$ plastic container sealed for ethylene accumulation at $20^{\circ} \mathrm{C}$ for $4-6$ hours at harvest and 1-2 hours after storage, avoiding excessive accumulation of carbon dioxide. Then, $1 \mathrm{~mL}$ of gas was taken from the headspace and injected into a gas chromatograph (Shimadzu GC 8A, Tokyo, Japan) equipped with a flame ionization detector (FID) and an alumina column (Supelco 80/100 Porapak column, $75 \mathrm{~cm} \times 5 \mathrm{~mm} \times 3 \mathrm{~mm}$ ). The oven and injector temperatures were $40^{\circ} \mathrm{C}$ and $150^{\circ} \mathrm{C}$, respectively [13]. The results were expressed as $\mu \mathrm{L}$ $\mathrm{C}_{2} \mathrm{H}_{4} \mathrm{~kg}^{-1} \mathrm{~h}^{-1}$. 


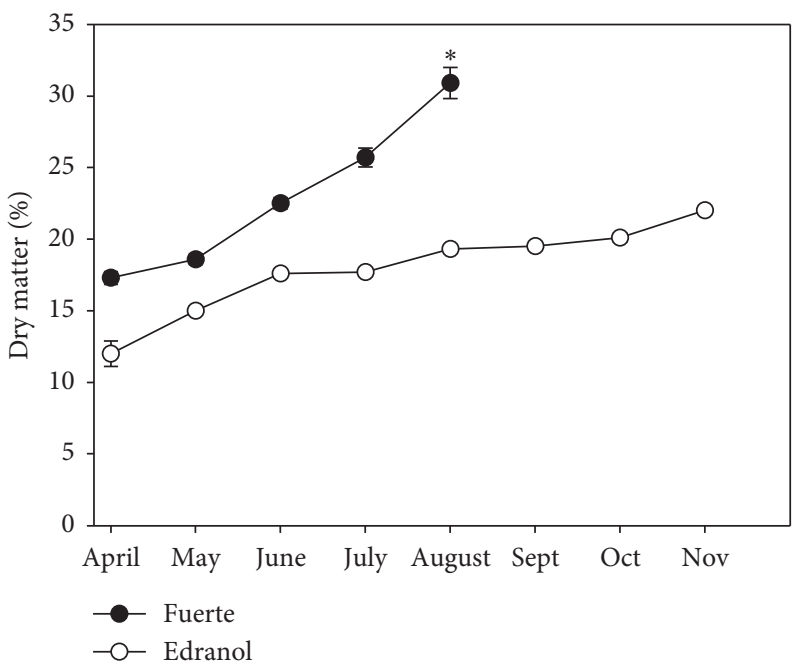

Figure 1: Dry matter content (\%) during development of avocado fruit in "Fuerte" and "Edranol" varieties. Asterisk (*) corresponds to harvest times. Each point corresponds to the mean \pm standard deviation.

TABLE 1: Quality variables at both harvest times in "Fuerte" and "Edranol" avocado fruit.

\begin{tabular}{|c|c|c|c|c|}
\hline & \multicolumn{2}{|c|}{ "Fuerte" } & \multicolumn{2}{|c|}{ "Edranol" } \\
\hline & Harvest I & Harvest II & Harvest I & Harvest II \\
\hline Harvest date & 06.27 .2018 & 08.30 .2018 & 09.10 .2018 & 11.05 .2018 \\
\hline Dry matter (DM) (\%) & $22.5 \pm 0.4$ & $30.9 \pm 1.1$ & $19.5 \pm 0.3$ & $22.0 \pm 0.3$ \\
\hline Firmness $(\mathrm{N})$ & $230.7 \pm 2.9$ & $247.5 \pm 4.4$ & $196.8 \pm 1.4$ & $209.3 \pm 2.5$ \\
\hline Weight (g) & $200.8 \pm 6.2$ & $195.8 \pm 10.4$ & $243.6 \pm 7.7$ & $234.3 \pm 8.2$ \\
\hline Polar diameter $(\mathrm{mm})$ & $108.7 \pm 2.1$ & $111.5 \pm 2.8$ & $115.5 \pm 1.7$ & $118.5 \pm 2.4$ \\
\hline Equatorial diameter (mm) & $61.9 \pm 0.9$ & $60.7 \pm 1.6$ & $67.4 \pm 0.8$ & $67.4 \pm 0.7$ \\
\hline
\end{tabular}

Values are the mean of 15 fruits \pm standard deviation.

The respiration rate was calculated based on the carbon dioxide produced by the fruit. The concentration of $\mathrm{CO}_{2}$ in the same plastic container was measured by taking $1 \mathrm{~mL}$ of gas from the headspace and injecting it into a gas analyzer (PBI-Dansensor Checkmate 9900, Ringsted, Denmark). The results were expressed in $\mathrm{mL} \mathrm{CO} \mathrm{kg}^{-1} \cdot \mathrm{h}^{-1}$.

2.3. RNA Isolation and cDNA Synthesis. Total RNA was isolated from the avocado samples using a modified hot borate method [15]. The quantity and quality of the RNA were assessed with a Qubit ${ }^{\circledR} 2.0$ fluorometer (Invitrogen ${ }^{\mathrm{TM}}$ by Life Technologies, Singapore), by measuring the A260/ 280 ratio and by electrophoresis on a $1.2 \%$ formaldehyde agarose gel. First-strand cDNA was obtained by reverse transcription with $2 \mu \mathrm{g}$ of total RNA as the template, using M-MLV reverse transcriptase (Promega, Madison, WI) and oligo dT primers according to standard procedures.

2.4. Real-Time Quantitative PCR Assays ( $q P C R$ ). Three ethylene synthesis genes were selected for characterization. The selection of genes was carried out based on a previously reported study [16, 17], and the genes were as follows: PamACO (1-aminocyclopropane-1-carboxylate oxidase, GenBank M32692.1); PamACS1 (1-aminocyclopropane-1carboxylate synthase 1, GenBank AF500119.1); and
PamACS2 (1-aminocyclopropane-1-carboxylate synthase 2, GenBank AF500120.1). The abundance of each transcript was analyzed by real-time PCR with a LightCycler real-time PCR system (Roche Diagnostics, Mannheim, Germany) using SYBR Green ${ }^{\mathrm{TM}}$ as a fluorescent dye to measure the amplified DNA products derived from the RNA. qPCR was performed on four replicates, and the gene expression values were normalized to the PamTCPB gene (T-complex protein 1 subunit beta, GenBank KT246107) [18]. Relative expression levels were determined using the comparative $\mathrm{Ct}$ method of Pfaffl [19], which was designed to measure the accumulation of mRNA relative to that of a constitutively expressed reference gene. Primers were designed using the Primer Premier 5.0 software package (Premier Biosoft International, Palo Alto, CA) and were synthesized by IDT (Integrated DNA Technologies). The primer sequences used in this study were as follows: PamACO forward 5'GTATTCGAGGACTACATGAATCTGT3', PamACO reverse 5'CCCTTTAGGTGGTAATGGGACT3', PamACS1 forward 5'GGGGAGATAGCACTTTGGCGT3', PamACS1 reverse $5^{\prime}$ CCTCCTTCATGGTCTCATCGTC3', PamACS2 forward 5'AGTGGTGGAGCGACAGGAGCA3', PamACS2 reverse 5'ACAGACGACTGGAATCAATTGGACT3', PamTCBP forward $5^{\prime}$ ATGATTGTTGGTATCGCCTGGA $\mathrm{G3}^{\prime}$, Pam $\mathrm{PCPB}$ reverse $5^{\prime}$ ATGATTGTTGGTATCGCCTG GAG 3'. 
2.5. Fruit Quality Parameters. At each sampling time, evaluation of quality attributes focused on the main factors, limiting quality in both varieties, i.e., pulp softening and the incidence of external and internal disorders. Flesh firmness was measured with a penetrometer equipped with a 4 - or 8-mm plunger tip; two measurements were made in each fruit side after removal of the epidermis [18]. Firmness measurements were performed at harvest, after cold storage and at the ready-to-eat stage. Fruit weight loss was measured in twelve avocados per treatment. Samples were weighed at harvest and after RA and CA storage, and the difference between them was considered the total weight loss and expressed as the percentage of loss from the initial weight according to the official method of the AOAC (1994).

For external (peel browning) and internal (gray pulp and vascular browning) damage, suitable hedonic scales for each disorder, ranging from 1 to 5 (1: no occurrence, 2: slight, 3: moderate, 4: moderately severe, and 5: severe) were used [18]. Results are reported as incidence (\%) of the disorder. In "Fuerte" avocados, pulp spot damage was also assessed visually using a hedonic scale from 1 (no occurrence) to 4 (severe).

In terms of fruit damage by decay, the presence or absence of stem end and body rot were evaluated, expressing the results as percentage of incidence. Skin color development is an important quality attribute in avocado, especially in "Hass." However, in this work, we did not analyze skin color because in preliminary studies carried out in our laboratory, differences in color were not observed during cold storage and shelf life.

2.6. Experimental Design and Statistical Analysis. A threeway factorial analysis of variance (ANOVA) was carried out to test the significance of the effects of treatment (RA, CA, and $1-\mathrm{MCP})$, storage time (30 and $50 \mathrm{~d}$ ), harvest (early and advanced maturity stages), and their interaction. Data in percentages were first converted by arcsine transformation prior to analysis, with nontransformed values used for presentation in figures. The means were separated with a least significant difference (LSD) test at 5\% significance using the statistical software InfoStat (version 2015, Universidad Nacional de Córdoba, Argentina). The post hoc analysis was made only when the effects of interactions and factors were significant, and nonsignificant data were not considered for the means test.

\section{Results and Discussion}

3.1. Ethylene Production and Respiration Rates. The ethylene production rates in "Fuerte" and "Edranol" stored under RA were higher than those in fruit stored under CA and treated with 1-MCP. This difference was observed at both maturity stages (Tables 2 and 3, for "Fuerte" and "Edranol," respectively). Immediately after $50 \mathrm{~d}$ of storage under RA, the ethylene production rate in "Edranol" was three to five times higher than that in fruit stored for $30 \mathrm{~d}$ from both harvests (Table 3), mainly due to the advanced ripening process observed in other avocado cultivars [20, 21].
Additionally, no detectable ethylene production was observed in fruit stored under CA in either variety (Tables 2 and 3). During shelf life at $20^{\circ} \mathrm{C}$, ethylene production rates increases in CA and 1-MCP-treated avocados of both varieties, but these increases did not reach the levels observed in fruit under RA until the ready-to-eat stage (data not shown).

In "Fuerte," the $\mathrm{CO}_{2}$ production rate at harvest was similar at both maturity stages. As expected for avocados after storage, the respiratory rate increased in fruit stored under RA, being higher in fruit with a lower DM content (early maturity stage), which was probably associated with a preclimacteric stage. In general, "Fuerte" avocados under CA, and especially under 1-MCP application, had lower respiratory rates than fruit stored under RA, especially fruit harvested at an earlier maturity stage, at both storage time (30 and $50 \mathrm{~d}$ ) (Table 2). A similar effect was also observed by Russo [2] in "Fuerte" when stored in modified atmosphere packaging with $6 \% \mathrm{CO}_{2}$ and $4 \% \mathrm{O}_{2}$, the same gas levels used in our study. Under RA storage, "Edranol" avocado showed an increase in respiration rate as storage time progressed (Table 3). Immediately after storage, only 1-MCP-treated fruit showed lower $\mathrm{CO}_{2}$ production rates for both periods than those stored under RA. Lemmer et al. [11] observed that the respiration rate in "Edranol" fruit stored in RA was higher than that in fruit stored under CA or treated with 1-MCP, with the latter being the most effective in reducing the respiratory rate. In fruit harvested at an advanced maturity stage, avocados stored under RA showed the highest $\mathrm{CO}_{2}$ production (Table 3 ).

3.2. Expression patterns of Genes Involved in Ethylene Biosynthesis. The ethylene synthesis pathway is well established in higher plants [22], and regulatory control is achieved at two steps: the formation of 1-aminocyclopropane-1-carboxylic acid (ACC) from S-adenosyl-1Met and the conversion of this intermediate to ethylene [23]. The first step is catalyzed by the enzyme ACC synthase (ACS) and the second by ACC oxidase (ACO). Both enzymes are encoded by multigene families that generate multiple points of control at which ethylene synthesis may be regulated. In this work, the genes that encode PamACO, PamACS1, and PamACS2 and their expression patterns were analyzed in "Fuerte" and "Edranol" avocado by semiquantitative qPCR. For all the genes in both varieties, an increase in expression level was observed after the cold storage period (Tables 4 and 5); this result was similar to that described by Hershkovitz et al. [17], which showed that low temperature during storage stimulates ACO and ACS gene expression in apple and pear, and chilling temperatures in the orchard induced avocado fruit ripening with increased ethylene biosynthesis.

In fruit from the early maturity stage, PamACO did not show great changes in expression after 30 or $50 \mathrm{~d}$ of RA storage in either variety (Tables 4 and 5). "Fuerte" avocados stored under RA showed higher expression than those stored under CA or treated with 1-MCP (Table 4); thus, higher expression was mainly associated with advancement of fruit 
TABLE 2: Ethylene production and respiration rates immediately after 30 and 50 days storage at $5^{\circ} \mathrm{C}$ in "Fuerte" avocado.

\begin{tabular}{|c|c|c|c|c|}
\hline Harvest & Storage time (days) & Treatment & $\begin{array}{c}\text { Ethylene production }\left(\mu \mathrm{LC}_{2} \mathrm{H}_{4} \mathrm{~kg}^{-1} \mathrm{~h}^{-1}\right) \\
\text { Mean } \pm \text { s.d }\end{array}$ & $\begin{array}{c}\text { Respiration rates }\left(\mathrm{mLCO}_{2} \mathrm{~kg}^{-1} \mathrm{~h}^{-1}\right) \\
\text { Mean } \pm \text { s.d }\end{array}$ \\
\hline \multirow{7}{*}{ I } & \multirow[t]{2}{*}{0} & Control & $0.05 \pm 0.11$ & $36.7 \pm 3.59$ \\
\hline & & RA & $3.68 \pm 3.29^{\mathrm{b}}$ & $72.7 \pm 46.9^{\mathrm{b}}$ \\
\hline & \multirow[t]{2}{*}{$30 \mathrm{~d}$} & CA & $0.00 \pm 0.00^{\mathrm{a}}$ & $34.4 \pm 3.49^{\mathrm{a}}$ \\
\hline & & $\mathrm{RA}+1-\mathrm{MCP}$ & $0.31 \pm 0.59^{\mathrm{a}}$ & $21.0 \pm 3.22^{\mathrm{a}}$ \\
\hline & \multirow{3}{*}{$50 \mathrm{~d}$} & RA & $6.34 \pm 4.87^{\mathrm{b}}$ & $72.4 \pm 4.95^{\mathrm{b}}$ \\
\hline & & CA & $0.00 \pm 0.00^{\mathrm{a}}$ & $36.3 \pm 2.93^{\mathrm{a}}$ \\
\hline & & $\mathrm{RA}+1-\mathrm{MCP}$ & $0.83 \pm 1.30^{\mathrm{a}}$ & $27.7 \pm 5.18^{\mathrm{a}}$ \\
\hline \multirow{7}{*}{ II } & \multirow[t]{2}{*}{0} & Control & $0.00 \pm 0.00$ & $43.9 \pm 11.30$ \\
\hline & & RA & $2.93 \pm 2.48^{\mathrm{b}}$ & $43.3 \pm 7.07^{\mathrm{b}}$ \\
\hline & \multirow[t]{2}{*}{$30 \mathrm{~d}$} & CA & $0.00 \pm 0.00^{\mathrm{a}}$ & $37.9 \pm 9.59^{\mathrm{ab}}$ \\
\hline & & $\mathrm{RA}+1-\mathrm{MCP}$ & $0.00 \pm 0.00^{\mathrm{a}}$ & $31.3 \pm 7.07^{\mathrm{a}}$ \\
\hline & \multirow{3}{*}{$50 \mathrm{~d}$} & RA & $5.47 \pm 4.77^{\mathrm{b}}$ & $53.5 \pm 5.01^{\mathrm{b}}$ \\
\hline & & CA & $0.00 \pm 0.00^{\mathrm{a}}$ & $55.4 \pm 9.98^{\mathrm{b}}$ \\
\hline & & $\mathrm{RA}+1-\mathrm{MCP}$ & $1.39 \pm 0.89^{\mathrm{a}}$ & $32.1 \pm 6.16^{\mathrm{a}}$ \\
\hline \multicolumn{5}{|c|}{ Interaction } \\
\hline \multicolumn{2}{|c|}{ Treatment (A) } & & $<0.0001^{*}$ & $<0.0001^{*}$ \\
\hline \multicolumn{2}{|c|}{ Storage time (B) } & & $<0.0001^{*}$ & $0.0340^{*}$ \\
\hline \multicolumn{2}{|c|}{ Harvest (C) } & & $0.0102 *$ & 0.5261 \\
\hline \multicolumn{2}{|c|}{$A \times B$} & & 0.0647 & 0.6651 \\
\hline \multicolumn{2}{|l|}{$\mathrm{A} \times \mathrm{C}$} & & 0.6585 & $<0.0001^{*}$ \\
\hline \multicolumn{2}{|l|}{$\mathrm{B} \times \mathrm{C}$} & & 0.7817 & 0.2353 \\
\hline \multicolumn{2}{|l|}{$\mathrm{A} \times \mathrm{B} \times \mathrm{C}$} & & 0.8859 & 0.2764 \\
\hline
\end{tabular}

RA: regular air (85\% Relative humidity); CA: controlled atmosphere ( $\left.4 \% \mathrm{O}_{2}+6 \% \mathrm{CO}_{2}\right)$; 1-MCP: 1-methylcyclopropene (300ppb); s.d: standard deviation. I: Early maturity stage; II: advanced maturity stage. d: days at $5^{\circ} \mathrm{C}$. Each value is the mean of ten fruits that were individually measured. Different letters within each storage time and harvest represents significant differences at $p \leq 0.05$ as determined by the LSD test. Factor: Treatment: RA, CA, RA + 1-MCP. Storage time: fruits storage for 30 and 50 days at $5^{\circ} \mathrm{C}$. Harvest: two harvest, early and advanced maturity stage. ${ }^{*}$ Factor significant, $\mathrm{p}<0.05$.

TABLE 3: Ethylene production and respiration rates immediately after 30 and 50 days storage at $5^{\circ} \mathrm{C}$ in "Edranol" avocado.

\begin{tabular}{|c|c|c|c|c|}
\hline Harvest & Storage time (days) & Treatment & $\begin{array}{c}\text { Ethylene production }\left(\mu \mathrm{LC}_{2} \mathrm{H}_{4} \mathrm{~kg}^{-1} \mathrm{~h}^{-1}\right) \\
\text { Mean } \pm \text { s.d }\end{array}$ & $\begin{array}{c}\text { Respiration rates }\left(\mathrm{mLCO}_{2} \mathrm{~kg}^{-1} \mathrm{~h}^{-1}\right) \\
\text { Mean } \pm \text { s.d }\end{array}$ \\
\hline \multirow{7}{*}{ I } & \multirow[t]{2}{*}{0} & Control & $0.00 \pm 0.0$ & $41.0 \pm 5.30$ \\
\hline & & RA & $8.82 \pm 7.60^{\mathrm{b}}$ & $34.5 \pm 7.70^{\mathrm{c}}$ \\
\hline & \multirow[t]{3}{*}{$30 \mathrm{~d}$} & CA & $0.00 \pm 0.00^{\mathrm{a}}$ & $28.3 \pm 2.60^{\mathrm{b}}$ \\
\hline & & $\mathrm{RA}+1-\mathrm{MCP}$ & $0.18 \pm 0.36^{\mathrm{a}}$ & $19.1 \pm 5.40^{\mathrm{a}}$ \\
\hline & & RA & $50.3 \pm 22.0^{\mathrm{b}}$ & $70.9 \pm 6.90^{c}$ \\
\hline & \multirow[t]{2}{*}{$50 \mathrm{~d}$} & $\mathrm{CA}$ & $0.21 \pm 0.33^{\mathrm{a}}$ & $37.5 \pm 7.70^{\mathrm{b}}$ \\
\hline & & $\mathrm{RA}+1-\mathrm{MCP}$ & $0.53 \pm 0.51^{\mathrm{a}}$ & $25.4 \pm 4.70^{\mathrm{a}}$ \\
\hline \multirow{7}{*}{ II } & \multirow[t]{2}{*}{0} & Control & $0.610 \pm 1.050$ & $24.6 \pm 3.30$ \\
\hline & & RA & $11.4 \pm 5.02^{\mathrm{b}}$ & $55.8 \pm 15.0^{\mathrm{c}}$ \\
\hline & \multirow[t]{2}{*}{$30 \mathrm{~d}$} & CA & $0.000 \pm 0.000^{\mathrm{a}}$ & $41.3 \pm 2.60^{\mathrm{b}}$ \\
\hline & & $\mathrm{RA}+1-\mathrm{MCP}$ & $0.230 \pm 0.240^{\mathrm{a}}$ & $31.4 \pm 4.20^{\mathrm{a}}$ \\
\hline & \multirow{3}{*}{$50 \mathrm{~d}$} & RA & $29.5 \pm 11.40^{\mathrm{b}}$ & $71.1 \pm 14.0^{\mathrm{c}}$ \\
\hline & & CA & $0.000 \pm 0.000^{\mathrm{a}}$ & $49.7 \pm 7.30^{\mathrm{b}}$ \\
\hline & & $\mathrm{RA}+1-\mathrm{MCP}$ & $1.510 \pm 0.850^{\mathrm{a}}$ & $36.8 \pm 2.60^{\mathrm{a}}$ \\
\hline \multicolumn{5}{|c|}{ Interaction } \\
\hline \multicolumn{2}{|c|}{ Treatment (A) } & & $<0.0001^{*}$ & $<0.0001^{*}$ \\
\hline \multicolumn{2}{|c|}{ Storage time (B) } & & $<0.0001^{*}$ & $<0.0001^{*}$ \\
\hline \multicolumn{2}{|c|}{ Harvest (C) } & & 0.0501 & $<0.0001^{*}$ \\
\hline \multicolumn{2}{|c|}{$\mathrm{A} \times \mathrm{B}$} & & $<0.0001^{*}$ & 0.8698 \\
\hline \multicolumn{2}{|l|}{$\mathrm{A} \times \mathrm{C}$} & & $0.0133^{*}$ & 0.8698 \\
\hline \multicolumn{2}{|l|}{$\mathrm{B} \times \mathrm{C}$} & & $0.0116^{*}$ & $0.0118^{*}$ \\
\hline \multicolumn{2}{|l|}{$\mathrm{A} \times \mathrm{B} \times \mathrm{C}$} & & $0.0011^{*}$ & $0.0075^{*}$ \\
\hline
\end{tabular}

RA: regular air (85\% Relative humidity); CA: controlled atmosphere $\left(4 \% \mathrm{O}_{2}+\times 6 \% \mathrm{CO}_{2}\right)$; 1-MCP: 1-methylcyclopropene (300 ppb); s.d: standard deviation. I: early maturity stage; II: advanced maturity stage. d: days at $5^{\circ} \mathrm{C}$. Each value is the mean of ten fruits that were individually measured. Different letters within each storage time and harvest represents significant differences at $p \leq 0.05$ as determined by the LSD test. Factor: treatment: RA, CA RA + 1-MCP. Storage time: fruits storage for 30 and 50 days at $5^{\circ} \mathrm{C}$. Harvest: two harvest, early and advanced maturity stage. ${ }^{*}$ Factor significant, $p<0.05$. 
TABle 4: Gene expression analysis of PamACO, PamACS1, and PamACS2 transcript in "Fuerte" avocado under different storage conditions for 30 and 50 days at $5^{\circ} \mathrm{C}$. Fruit was harvested at two maturity stages.

\begin{tabular}{|c|c|c|c|c|c|c|c|c|c|c|c|}
\hline \multirow{2}{*}{ Harvest } & \multirow{2}{*}{ Storage time (days) } & \multirow{2}{*}{ Treatment } & \multicolumn{3}{|c|}{$\mathrm{ACO}$} & \multicolumn{3}{|c|}{ ACS1 } & \multicolumn{3}{|c|}{ ACS2 } \\
\hline & & & Mean & & s.d & Mean & & s.d & Mean & & s.d \\
\hline \multirow{7}{*}{$I$} & 0 & Control & 0.02 & \pm & 0.09 & 0.020 & \pm & 0.01 & 1.63 & \pm & 2.05 \\
\hline & & RA & 9.07 & \pm & $4.31^{\mathrm{b}}$ & 9.85 & \pm & $7.42^{\mathrm{b}}$ & 1.50 & \pm & $0.48^{\mathrm{a}}$ \\
\hline & $30 \mathrm{~d}$ & CA & 0.79 & \pm & $0.41^{\mathrm{a}}$ & 0.83 & \pm & $0.40^{\mathrm{a}}$ & 0.81 & \pm & $0.41^{\mathrm{a}}$ \\
\hline & & $\mathrm{RA}+1-\mathrm{MCP}$ & 0.41 & \pm & $0.30^{\mathrm{a}}$ & 0.57 & \pm & $0.63^{\mathrm{a}}$ & 0.68 & \pm & $0.48^{\mathrm{a}}$ \\
\hline & $50 \mathrm{~d}$ & RA & 9.36 & \pm & $3.05^{\mathrm{b}}$ & 12.70 & \pm & $5.37^{\mathrm{b}}$ & 1.20 & \pm & $0.56^{\mathrm{a}}$ \\
\hline & & CA & 2.03 & \pm & $0.70^{\mathrm{a}}$ & 1.60 & \pm & $0.38^{\mathrm{a}}$ & 4.77 & \pm & $6.54^{\mathrm{a}}$ \\
\hline & & $\mathrm{RA}+1-\mathrm{MCP}$ & 2.51 & \pm & $1.94^{\mathrm{a}}$ & 3.44 & \pm & $3.56^{\mathrm{a}}$ & 0.65 & \pm & $0.19^{\mathrm{a}}$ \\
\hline \multirow{7}{*}{ II } & 0 & Control & 0.01 & \pm & 0.001 & 0.01 & \pm & 0.00 & 0.00 & \pm & 0.00 \\
\hline & & RA & 9.10 & \pm & $0.80^{\mathrm{b}}$ & 8.10 & \pm & $4.10^{\mathrm{b}}$ & 0.00 & \pm & $0.00^{\mathrm{a}}$ \\
\hline & $30 \mathrm{~d}$ & CA & 0.50 & \pm & $0.18^{\mathrm{a}}$ & 0.73 & \pm & $0.06^{\mathrm{a}}$ & 0.00 & \pm & $0.00^{\mathrm{a}}$ \\
\hline & & $\mathrm{RA}+1-\mathrm{MCP}$ & 2.18 & \pm & $0.86^{\mathrm{a}}$ & 1.89 & \pm & $0.73^{\mathrm{a}}$ & 0.00 & \pm & $0.00^{\mathrm{a}}$ \\
\hline & $50 \mathrm{~d}$ & RA & 2.40 & \pm & $1.93^{\mathrm{a}}$ & 3.62 & \pm & $3.12^{\mathrm{a}}$ & 0.00 & \pm & $0.00^{\mathrm{a}}$ \\
\hline & & CA & 2.00 & \pm & $1.51^{\mathrm{a}}$ & 1.60 & \pm & $0.66^{\mathrm{a}}$ & 0.00 & \pm & $0.00^{\mathrm{a}}$ \\
\hline & & $\mathrm{RA}+1-\mathrm{MCP}$ & 8.40 & \pm & $2.50^{\mathrm{b}}$ & 14.17 & \pm & $3.92^{\mathrm{b}}$ & 0.00 & \pm & $0.00^{\mathrm{a}}$ \\
\hline \multicolumn{12}{|l|}{ Interaction } \\
\hline Treatment (A) & & & & $<0.0001^{*}$ & & & $<0.0001^{*}$ & & & 0.4386 & \\
\hline Storage time (B) & & & & 0.0751 & & & $0.0083^{*}$ & & & 0.2649 & \\
\hline Harvest (C) & & & & 0.6316 & & & 0.8529 & & & $0.0023^{*}$ & \\
\hline $\mathrm{A} \times \mathrm{B}$ & & & & $0.001^{*}$ & & & $0.0012^{*}$ & & & 0.2004 & \\
\hline$A \times C$ & & & & $<0.0001^{*}$ & & & $0.0001^{*}$ & & & 0.4386 & \\
\hline $\mathrm{B} \times \mathrm{C}$ & & & & 0.9149 & & & 0.6898 & & & 0.2649 & \\
\hline $\mathrm{A} \times \mathrm{B} \times \mathrm{C}$ & & & & $0.0228^{*}$ & & & $0.0023^{*}$ & & & 0.2004 & \\
\hline
\end{tabular}

RA: regular air ( $85 \%$ relative humidity); CA: controlled atmosphere $\left(4 \% \mathrm{O}_{2}+6 \% \mathrm{CO}_{2}\right)$; 1-MCP: 1-methylcyclopropene (300 ppb); s.d: standard deviation. I: early maturity stage; II: advanced maturity stage. d: days at $5^{\circ} \mathrm{C}$. The accumulation of PamACO, PamACS1, and PamACS2 was measured by qPCR in fruit at harvest and after cold storage in different treatments. Expression was normalized with the house-keeping gene PamTCPB. Each value corresponds to mean of three fruits \pm sd. Different letters within each storage time and harvest represents significant differences at $P \leq 0.05$ as determined by the LSD test. Factor: treatment: RA, CA, and RA + 1-MCP. Storage time: fruits storage for 30 and 50 days at $5{ }^{\circ} \mathrm{C}$. Harvest: two harvest, early and advanced maturity stages. ${ }^{*}$ Factor significant, $P \leq 0.05$.

ripening stage. An increase in PamACO expression levels was observed after $50 \mathrm{~d}$ of storage under CA and 1-MCP application in "Fuerte" from both harvests (Table 4). In "Edranol, "a significant increase in the expression level of PamACS1 was observed after $50 \mathrm{~d}$ of storage under RA in the early maturity stage (Table 5), and these changes were concomitant with decreased flesh firmness (Figure 2(c)) and increased ethylene production (Table 3). These results were different from those observed in "Fuerte" which did not show any change in expression level independent of the storage period (Table 4). Under all storage conditions, low expression levels were observed for PamACS2 in "Fuerte" and "Edranol, "without significant differences between treatments or storage periods (Tables 4 and 5). The PamACO and PamACS1 transcript levels showed expression profiles similar to those described by Hershkovitz et al. [16], being very low at harvest, with a major increase after storage associated with climacteric ethylene production. The difference in expression profiles observed between the maturity stages could be associated mainly with fruit heterogeneity caused by the long flowering period, which generates high variability in fruit age at harvest [24].

3.3. Flesh Firmness. "Fuerte" avocados at both maturity stages stored under RA showed a sharp decrease in flesh firmness during storage, from $230 \mathrm{~N}$ at harvest to $70 \mathrm{~N}$ and
$20 \mathrm{~N}$ after 30 and $50 \mathrm{~d}$, respectively (Figures 2(a) and 2(b)). Fruits stored under CA maintained firmness levels close to those observed at harvest, independent of the storage period or maturity stage (Figures 2(a) and 2(b)). Similar effects were reported in "Hass" avocado, where the use of low oxygen and high carbon dioxide significantly reduced softening during storage [25]. Flesh firmness in the 1-MCP-treated fruit was similar to that of CA fruit after $30 \mathrm{~d}$ of storage for early harvested fruit but showed a decrease after $50 \mathrm{~d}$ of storage. Both CA-treated and 1-MCP-treated fruit showed higher firmness levels than fruit stored under RA (Figures 2(a) and 2(b)).

For "Edranol" avocados, the flesh firmness of the fruit stored under RA showed a significant decrease from $200 \mathrm{~N}$ at harvest to $20 \mathrm{~N}$ after $50 \mathrm{~d}$ of storage (Figures 2(c) and 2(d)). Fruits stored under CA and 1-MCP application maintained similar flesh firmness levels to those measured at harvest after both storage times ( 30 and $50 \mathrm{~d}$ ), independent of maturity stage (Figures 2(c) and 2(d)). The firmness retention in both cultivars was significantly enhanced in response to 1-MCP treatment, mainly due to the high ethylene dependency of this quality attribute in avocado [13]. Similar effects of 1-MCP in delaying avocado fruit softening have been observed for the "Hass" cultivar $[8,26]$. The differences observed between "Fuerte" and "Edranol" avocado could be due to differences in developmental stage, as shown by the DM content, because "Edranol" was harvested with lower 
TABle 5: Gene expression analysis of PamACO, PamACS1, and PamACS2 transcript in avocado "Edranol" under different storage conditions for 30 and 50 days at $5^{\circ} \mathrm{C}$. Fruit was harvested at two maturity stages.

\begin{tabular}{|c|c|c|c|c|c|c|c|c|c|c|c|}
\hline \multirow{2}{*}{ Harvest } & \multirow{2}{*}{ Storage time (days) } & \multirow{2}{*}{ Treatment } & \multicolumn{3}{|c|}{$\mathrm{ACO}$} & \multicolumn{3}{|c|}{ ACS1 } & \multicolumn{3}{|c|}{ ACS2 } \\
\hline & & & Mean & & s.d & Mean & & s.d & Mean & & s.d \\
\hline \multirow{7}{*}{$I$} & 0 & Control & 0.03 & \pm & 0.01 & 0.01 & \pm & 0.01 & 2.99 & \pm & 1.24 \\
\hline & & RA & 13.59 & \pm & $6.60^{\mathrm{b}}$ & 6.63 & \pm & $2.63^{\mathrm{b}}$ & 1.10 & \pm & $0.24^{\mathrm{a}}$ \\
\hline & $30 \mathrm{~d}$ & $\mathrm{CA}$ & 0.27 & \pm & $0.04^{\mathrm{a}}$ & 0.26 & \pm & $0.02^{\mathrm{a}}$ & 0.53 & \pm & $0.25^{\mathrm{a}}$ \\
\hline & & $\mathrm{RA}+1-\mathrm{MCP}$ & 0.63 & \pm & $0.13^{\mathrm{a}}$ & 1.05 & \pm & $0.19^{\mathrm{a}}$ & 0.60 & \pm & $0.30^{\mathrm{a}}$ \\
\hline & $50 \mathrm{~d}$ & RA & 17.45 & \pm & $2.82^{\mathrm{b}}$ & 27.6 & \pm & $0.93^{\mathrm{b}}$ & 1.04 & \pm & $0.20^{\mathrm{a}}$ \\
\hline & & $\mathrm{CA}$ & 1.18 & \pm & $0.18^{\mathrm{a}}$ & 0.90 & \pm & $0.20^{\mathrm{a}}$ & 0.88 & \pm & $0.44^{\mathrm{a}}$ \\
\hline & & $\mathrm{RA}+1-\mathrm{MCP}$ & 1.35 & \pm & $0.17^{\mathrm{a}}$ & 3.02 & \pm & $0.34^{\mathrm{a}}$ & 0.73 & \pm & $0.25^{\mathrm{a}}$ \\
\hline \multirow{7}{*}{ II } & 0 & Control & 0.01 & \pm & 0.01 & 0.11 & \pm & 0.04 & 0.87 & \pm & 0.84 \\
\hline & & RA & 15.97 & \pm & $6.60^{\mathrm{b}}$ & 14.28 & \pm & $1.83^{\mathrm{b}}$ & 0.77 & \pm & $0.47^{\mathrm{a}}$ \\
\hline & $30 \mathrm{~d}$ & CA & 0.24 & \pm & $0.07^{\mathrm{a}}$ & 0.77 & \pm & $0.27^{\mathrm{a}}$ & 0.74 & \pm & $0.55^{\mathrm{a}}$ \\
\hline & & $\mathrm{RA}+1-\mathrm{MCP}$ & 0.77 & \pm & $0.14^{\mathrm{a}}$ & 0.88 & \pm & $0.10^{\mathrm{a}}$ & 0.90 & \pm & $0.62^{\mathrm{a}}$ \\
\hline & $50 \mathrm{~d}$ & RA & 9.04 & \pm & $1.62^{\mathrm{b}}$ & 4.67 & \pm & $0.98^{\mathrm{b}}$ & 2.59 & \pm & $1.57^{\mathrm{a}}$ \\
\hline & & $\mathrm{CA}$ & 0.88 & \pm & $0.41^{\mathrm{a}}$ & 0.03 & \pm & $0.01^{\mathrm{a}}$ & 1.12 & \pm & $0.73^{\mathrm{a}}$ \\
\hline & & $\mathrm{RA}+1-\mathrm{MCP}$ & 3.55 & \pm & $1.76^{\mathrm{a}}$ & 7.37 & \pm & $2.56^{\mathrm{c}}$ & 2.45 & \pm & $1.88^{\mathrm{a}}$ \\
\hline \multicolumn{12}{|l|}{ Interaction } \\
\hline Treatment (A) & & & & $<0.0001^{*}$ & & & $<0.0001^{*}$ & & & 0.6029 & \\
\hline Storage time (B) & & & & 0.3587 & & & $0.0386^{*}$ & & & $0.0137^{*}$ & \\
\hline Harvest (C) & & & & 0.9717 & & & 0.7575 & & & $0.0385^{*}$ & \\
\hline $\mathrm{A} \times \mathrm{B}$ & & & & 0.7862 & & & 0.266 & & & 0.7672 & \\
\hline $\mathrm{A} \times \mathrm{C}$ & & & & 0.6695 & & & 0.2665 & & & 0.1003 & \\
\hline $\mathrm{B} \times \mathrm{C}$ & & & & $0.0305^{*}$ & & & $0.0022^{*}$ & & & 0.0609 & \\
\hline $\mathrm{A} \times \mathrm{B} \times \mathrm{C}$ & & & & $0.0009^{*}$ & & & $<0.0001^{*}$ & & & 0.3244 & \\
\hline
\end{tabular}

RA: regular air (85\% relative humidity); CA: controlled atmosphere $\left(4 \% \mathrm{O}_{2}+6 \% \mathrm{CO}_{2}\right)$; 1-MCP: 1-methylcyclopropene (300 ppb); s.d: standard deviation. I: early maturity stage; II: advanced maturity stage; d: days at $5^{\circ} \mathrm{C}$. The accumulation of PamACO, PamACS1, and PamACS 2 was measured by qPCR in fruit at harvest and after cold storage in different treatments. Expression was normalized with the house-keeping gene PamTCPB. Each value corresponds to mean of three fruits \pm sd. Different letters within each storage time and harvest represent significant differences at $P \leq 0.05$ as determined by the LSD test. Factor: treatment: RA, CA, and RA + 1-MCP. Storage time: fruits storage for 30 and 50 days at $5^{\circ} \mathrm{C}$. Harvest: two harvest, early and advanced maturity stages. ${ }^{*}$ Factor significant, $P<0.05$.

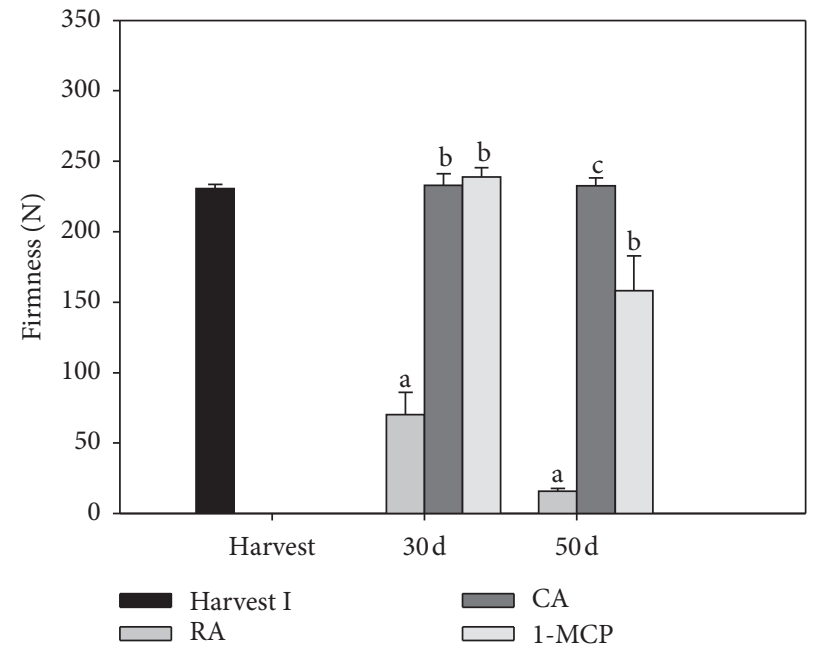

(a)

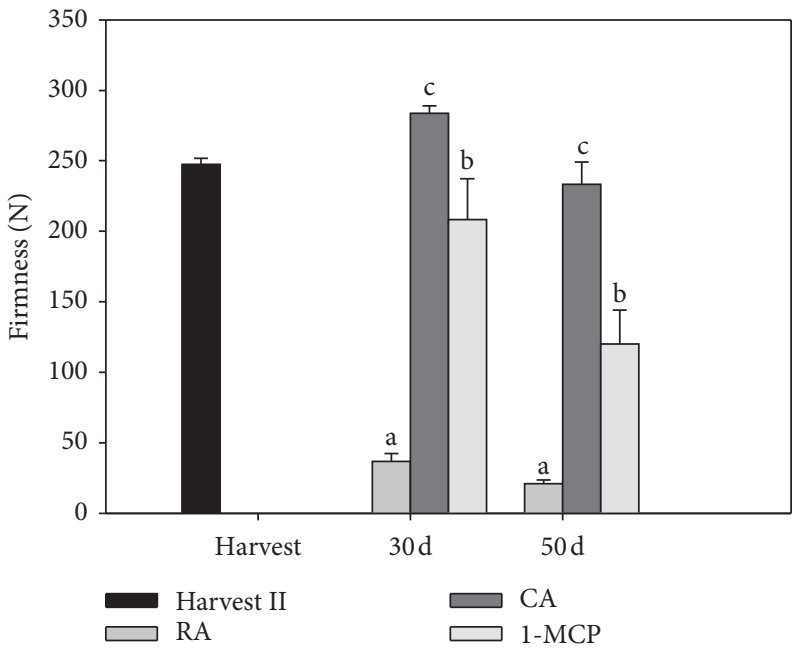

(b)

Figure 2: Continued. 


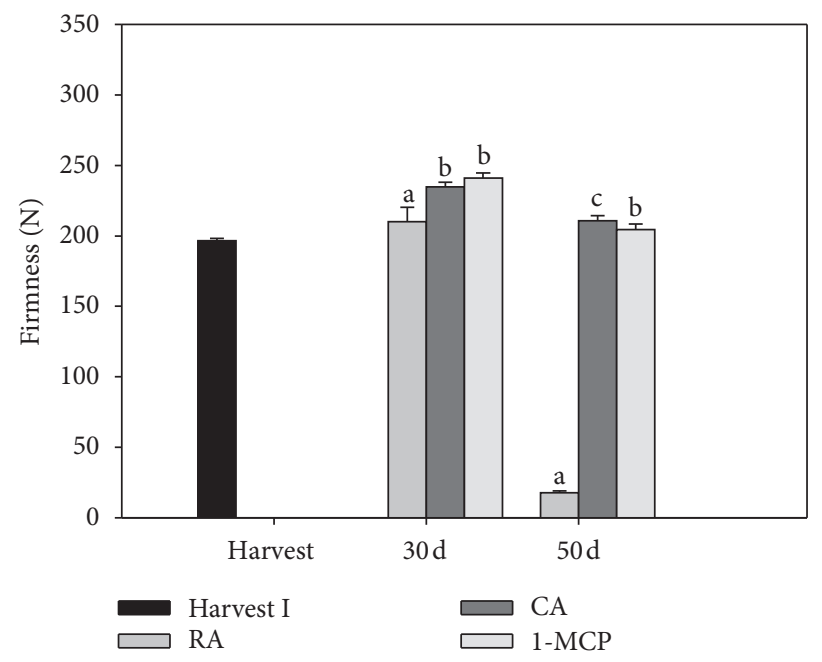

(c)

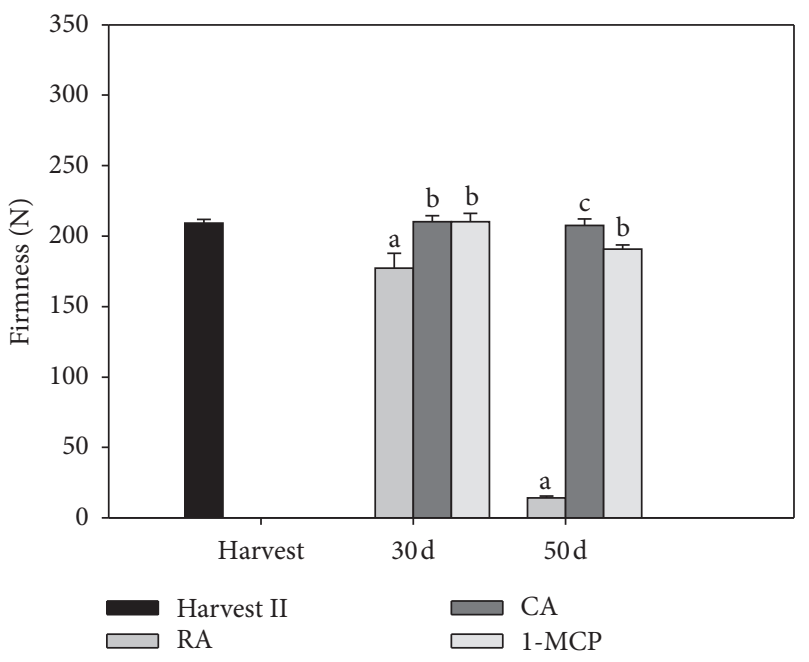

(d)

FIGURE 2: Flesh firmness immediately after storage at $5^{\circ} \mathrm{C}$ for 30 and 50 days. Each value is the mean of thirty-five fruits for treatment that was individually measured +/- standard errors. (a, c) Harvest I. (b, d) Harvest II. RA: regular air. CA: controlled atmospheres (4\% $\mathrm{O}_{2}$ and $6 \%$ $\mathrm{CO}_{2}$ ). Different letters within each storage period represent significant differences at $P \leq 0.05$ as determined by the LSD test. d: days.

DM than "Fuerte". After storage, "Fuerte" and "Edranol" avocado stored at $20^{\circ} \mathrm{C}$ under RA softened rapidly, reaching the ready-to-eat stage in 3 to $4 \mathrm{~d}$, depending on the storage length. Fruits stored under CA and treated with 1-MCP took 7 and $10 \mathrm{~d}$ to reach the ready-to-eat stage after cold storage (Table 6). Similar results have been reported for "Hass" avocados [13]. Fruits stored under RA at $5^{\circ} \mathrm{C}$ for 3.5 weeks began to soften during storage and reached the ready-to-eat stage a week later when exposed to $20^{\circ} \mathrm{C}$ [26]. Lemmer et al. [11] showed that the time required for fruit to reach the ready-to-eat stage after cold storage under RA was less than the time required under CA or 1-MCP application; in "Fuerte" and "Hass" the difference was 2 to $3 \mathrm{~d}$. In other varieties, such as "Edranol," "Pinkerton," and "Ryan," fruit stored under RA took 3 to 5 -fold less time to reach the readyto-eat stage than CA or 1-MCP-treated fruit.

To understand the changes in avocado texture in 1MCP-treated fruit, previous results indicated that polygalacturonase (PG) activity correlated with a decrease in galacturonic acid in the avocado cell wall material. 1-MCP application modified the PG and pectin methyl esterase (PME) activities from those observed in untreated fruit, but when avocados were soft $(19.6 \mathrm{~N})$, the PG and PME activities were equal to those in control fruit not treated with 1-MCP $[12,13]$.

3.4. Weight Loss. In "Fuerte" avocado at the early maturity stage, the weight loss was no greater than 3.5\% in fruit stored for 30 or $50 \mathrm{~d}$, with lower levels obtained in fruit under CA with values close to $1 \%$ (Table 7). "Fuerte" avocado at the advanced maturity stage showed a greater weight loss than fruit at the early maturity stage $(23 \% \mathrm{DM})$ (Table 7$)$. In fruit stored for $30 \mathrm{~d}$, the weight loss was no greater than $3 \%$ in RA-treated and in 1-MCP-treated avocados; however, in fruit stored under CA, weight loss did not exceed $1 \%$. After
$50 \mathrm{~d}$ of storage, weight loss increased to higher than $5 \%$ in fruit under RA, and lower levels were maintained in fruit under CA or those treated with 1-MCP, which had weight losses of 1.8 and $4.8 \%$, respectively. Russo et al. [2] reported similar results in "Fuerte" avocados studying different concentrations of $\mathrm{O}_{2}$ and $\mathrm{CO}_{2}$ at low temperature and determined that $7 \% \mathrm{CO}_{2}$ and $4 \% \mathrm{O}_{2}$ were the most effective concentrations for minimizing weight loss. The weight loss in avocado is low under ideal storage conditions with high RH [27]. In "Edranol," weight loss was similar to that observed in "Fuerte" with respect to differences among treatments and storage periods. Fruit from the first harvest showed a higher weight loss than fruit from the advanced maturity stage. The lower weight loss in the CA treatment could be because the $\mathrm{RH}$ in the CA chamber remains higher than that in RA storage, reducing the vapor pressure deficit. In fact, the use of plastic films and refrigeration is among the suggested strategies to reduce weight loss in several fruits $[28,29]$. The difference in weight loss observed in fruit stored in RA could be due to the major effect of 1-MCP in delaying the ripening process compared with that in the untreated fruit.

3.5. External Damage. In "Fuerte," the incidence of external damage immediately after storage was observed in fruit at the early maturity stage and that stored under RA (Table 8). Fruit under CA did not show any symptoms associated with external damage, and 1-MCP-treated avocados showed a low incidence of damage after $50 \mathrm{~d}$ of storage (Table 8). However, after reaching the ready-to-eat stage at $20^{\circ} \mathrm{C}$, external damage showed an increase in both treatments, being higher in 1-MCP-treated fruit.

For "Edranol" avocado, this disorder was observed mainly in fruit under RA storage at both maturity stages at harvest. In early maturity fruit, $80 \%$ damage was observed 
TABLE 6: Days required for reaching a ready-to-eat stage during shelf life at $20^{\circ} \mathrm{C}$, after each storage period at $5^{\circ} \mathrm{C}$.

\begin{tabular}{lcccc}
\hline Treatment & & "Fuerte" & & "Edranol" \\
\hline RA 30d & Harvest I & Harvest II & 3 & Harvest I \\
CA 30d & 4 & 3 & 10 & 4 \\
1 -MCP 30d & 7 & 7 & 10 & 7 \\
\hline RA 50d & 10 & 8 & 3 & 1 \\
CA 50d & 3 & 7 & 7 & 4 \\
1-MCP 50d & 8 & 8 & 5 & 3 \\
\hline
\end{tabular}

Values are the mean of 75 fruit for treatment. d: days. CA: controlled atmosphere. RA: regulate air.

TABle 7: Weight loss (\%) in "Fuerte" and "Edranol" avocados after cold storage.

\begin{tabular}{lcccc}
\hline & & & & "Fdranol" \\
& Harvest I & Harvest II & Harvest I & Harvest II \\
\hline RA 30 d & $2.48 \pm 0.16$ & $3.07 \pm 0.16$ & $2.62 \pm 0.16$ & $1.44 \pm 0.16$ \\
CA 30 d & $0.84 \pm 0.05$ & $0.75 \pm 0.16$ & $1.13 \pm 0.14$ & $0.53 \pm 0.06$ \\
1 -MCP 30 d & $1.80 \pm 0.13$ & $2.91 \pm 0.16$ & $2.39 \pm 0.23$ & $1.76 \pm 0.18$ \\
RA 50 d & $3.41 \pm 0.47$ & $5.45 \pm 0.47$ & $5.39 \pm 0.20$ & $3.64 \pm 0.24$ \\
CA 50 d & $1.12 \pm 0.07$ & $1.89 \pm 0.04$ & $1.33 \pm 0.07$ & $1.04 \pm 0.08$ \\
1-MCP 50 d & $2.96 \pm 0.14$ & $4.83 \pm 0.21$ & $4.49 \pm 0.33$ & $2.99 \pm 0.27$ \\
\hline
\end{tabular}

Values are the mean of 20 fruits \pm standard deviation. d: days. CA: controlled atmosphere. RA: regulate air.

after $30 \mathrm{~d}$ of storage and then increased to levels close to $90 \%$ after $50 \mathrm{~d}$ at $5^{\circ} \mathrm{C}$ (Table 9). These values increased after shelf life, reaching almost $100 \%$. A similar pattern was observed in fruit at the advanced maturity stage, which showed an $80 \%$ incidence after $30 \mathrm{~d}$ of storage and close to $60 \%$ after $50 \mathrm{~d}$ (Table 9). As in "Fuerte" avocados, both technological approaches significantly reduced the incidence of external damage; this disorder appeared only after $50 \mathrm{~d}$ of storage in 1-MCP-treated avocados (Tables 8 and 9). Interestingly, in this variety, the benefits of CA treatment were observed until the advanced stage of ripening during shelf life exposure.

External damage in avocado has been associated with the development of physiological disorders, some of which are related to chilling injury at low temperature or fruit senescence [30]. These disorders may show up during cold storage, as observed in "Edranol" and "Fuerte" for RA storage or after reaching a ready-to-eat stage at room temperature, as shown in fruit stored under CA or treated with 1-MCP [31, 32]. Chilling injury symptoms during cold storage manifest in several ways, such as skin browning, internal browning, vascular browning, and increased pathogen susceptibility [33], and their relative importance depends on avocado variety, storage time, environmental conditions, and growing conditions and maturity stage, among factors.

3.6. Internal Damage. Gray pulp (a type of mesocarp discoloration) and vascular browning were the most important disorders observed in this study. Gray pulp is a diffuse area of discolored gray or gray/brown flesh with a poorly defined margin, usually starting at the bottom of the fruit adjacent to the seed and spreading upwards and outwards [34]. In this study, gray pulp was observed mainly in "Fuerte" immediately after storage, fruit from the early maturity stage stored for $30 \mathrm{~d}$ in RA showed an approximately $2 \%$ incidence that increased to $20 \%$ with extended storage (Table 8). Both CA-treated and 1MCP-treated fruit showed almost no damage immediately after storage but showed an increase in gray pulp after shelf life, especially in fruit harvested at the advanced maturity stage; the incidence reached 12 and 32\% after $50 \mathrm{~d}$ in 1-MCP-treated and CA stored avocados, respectively (Table 8). For the "Edranol" variety, a $20 \%$ incidence of gray pulp was observed in fruit at the advanced maturity stage stored for $50 \mathrm{~d}$ under RA, increasing to $55 \%$ after exposure at $20^{\circ} \mathrm{C}$ (Table 9). The CAtreated and 1-MCP-treated fruit did not show this disorder immediately after storage or at the ready-to-eat stage. Similar results have been reported in other varieties, such as "Ettinger," "Pinkerton" and "Hass" where 1-MCP was very effective in reducing gray pulp in all tested avocado cultivars $[8,26,35]$.

Vascular browning has been described as a brown vascular bundle discoloration that usually starts at the stem end or base of the fruit [34]. This disorder was observed mainly in fruit of the advanced maturity stage stored under RA, showing an incidence close to $17 \%$ in "Fuerte" (Table 8) and $11 \%$ in "Edranol" after $50 \mathrm{~d}$ of storage (Table 9) but increasing to levels close to $40-50 \%$ in both varieties during shelf life. Both CA-treated and 1-MCP-treated fruit of both varieties showed a reduced incidence of vascular browning at the end of storage and an increased incidence after exposure to $20^{\circ} \mathrm{C}$ (Tables 8 and 9 ).

Another physiological disorder observed in this research was pulp spot, which was mainly observed in "Fuerte."This disorder is characterized by black or gray spots along the margins of the vascular bundles, distributed randomly throughout the fruit. The incidence and severity of this disorder are strongly associated with cultural practices; mainly linked to calcium, potassium, and magnesium ratios; and highly variable across seasons [36]. The symptoms develop after storage and 
TABLE 8: Evaluation of physiological disorders in "Fuerte" avocado under different storage conditions for 30 and 50 days at $5^{\circ} \mathrm{C}$. RA: regular air (85\% relative humidity); CA: controlled atmosphere $\left(4 \% \mathrm{O}_{2}+6 \% \mathrm{CO}_{2}\right)$; 1-MCP: 1-methylcyclopropene (300 ppb); s.d: standard deviation. I: early maturity stage; II: advanced maturity stage. D: days at $5^{\circ} \mathrm{C}$. SL (shelf life): fruit storage at $20^{\circ} \mathrm{C}$. Fruit at SL was evaluated at the same flesh firmness level. Each value corresponds to mean of 35 fruits. Different letters within each storage time and harvest represent significant differences at as determined by the LSD test.

\begin{tabular}{|c|c|c|c|c|c|c|c|c|c|c|c|c|c|c|}
\hline \multirow{3}{*}{ Variable } & \multirow{3}{*}{ Harvest } & \multirow{3}{*}{ Treatment } & \multicolumn{12}{|c|}{ Storage time (days) } \\
\hline & & & \multicolumn{3}{|c|}{$30 \mathrm{~d}$} & \multicolumn{3}{|c|}{$30+S L$} & \multicolumn{3}{|c|}{$50 \mathrm{~d}$} & \multicolumn{3}{|c|}{$50+\mathrm{SL}$} \\
\hline & & & Mean & \pm & $\mathrm{SD}$ & Mean & & $\mathrm{SD}$ & Mean & & $\mathrm{SD}$ & Mean & & SD \\
\hline \multirow{6}{*}{$\begin{array}{l}\text { External damage } \\
(\%)\end{array}$} & \multirow{4}{*}{ I } & RA & 6.7 & \pm & $11.6^{\mathrm{a}}$ & 10.5 & \pm & $8.3^{\mathrm{b}}$ & 4.8 & \pm & $3.3^{\mathrm{b}}$ & 18.1 & \pm & $4.4^{\mathrm{b}}$ \\
\hline & & CA & 0.0 & \pm & $0.0^{\mathrm{a}}$ & 1.9 & \pm & $3.3^{\mathrm{ab}}$ & 0.0 & \pm & $0.0^{\mathrm{a}}$ & 3.8 & \pm & $1.7^{\mathrm{a}}$ \\
\hline & & $\mathrm{RA}+1-\mathrm{MCP}$ & 0.0 & \pm & $0.0^{\mathrm{a}}$ & 1.0 & \pm & $1.7^{\mathrm{a}}$ & 3.8 & \pm & $1.6^{\mathrm{b}}$ & 13.3 & \pm & $1.7^{\mathrm{b}}$ \\
\hline & & RA & 0.0 & \pm & $0.0^{\mathrm{a}}$ & 5.7 & \pm & $5.7^{\mathrm{a}}$ & 0.0 & \pm & $0.0^{\mathrm{a}}$ & 6.7 & \pm & $5.9^{\mathrm{a}}$ \\
\hline & \multirow[t]{2}{*}{ II } & CA & 0.0 & \pm & $0.0^{\mathrm{a}}$ & 7.6 & \pm & $3.3^{\mathrm{a}}$ & 1.9 & \pm & $3.3^{\mathrm{a}}$ & 9.5 & \pm & $6.6^{\mathrm{ab}}$ \\
\hline & & $\mathrm{RA}+1-\mathrm{MCP}$ & 0.0 & \pm & $0.0^{\mathrm{a}}$ & 23.8 & \pm & $3.3^{\mathrm{b}}$ & 3.8 & \pm & $6.6^{\mathrm{a}}$ & 24.8 & \pm & $9.2^{\mathrm{b}}$ \\
\hline \multirow{6}{*}{ Gray pulp (\%) } & \multirow{3}{*}{ I } & RA & 1.9 & \pm & $1.7^{\mathrm{b}}$ & 17.1 & \pm & $5.0^{\mathrm{b}}$ & 19.0 & \pm & $6.0^{\mathrm{b}}$ & 58.1 & \pm & $8.7^{\mathrm{b}}$ \\
\hline & & $\mathrm{CA}$ & 0.0 & \pm & $0.0^{\mathrm{a}}$ & 2.9 & \pm & $2.9^{\mathrm{a}}$ & 0.0 & \pm & $0.0^{\mathrm{a}}$ & 4.8 & \pm & $8.3^{\mathrm{a}}$ \\
\hline & & $\mathrm{RA}+1-\mathrm{MCP}$ & 0.0 & \pm & $0.0^{\mathrm{a}}$ & 0.0 & \pm & $0.0^{\mathrm{a}}$ & 5.7 & \pm & $7.6^{\mathrm{a}}$ & 6.7 & \pm & $6.6^{\mathrm{a}}$ \\
\hline & \multirow{3}{*}{ II } & RA & 9.5 & \pm & $8.7^{\mathrm{b}}$ & 38.1 & \pm & $6.0^{\mathrm{b}}$ & 24.8 & \pm & $1.7^{\mathrm{b}}$ & 63.8 & \pm & $7.2^{\mathrm{c}}$ \\
\hline & & $\mathrm{CA}$ & 0.0 & \pm & $0.0^{\mathrm{a}}$ & 2.9 & \pm & $0.0^{\mathrm{a}}$ & 0.0 & \pm & $0.0^{\mathrm{a}}$ & 32.4 & \pm & $9.2^{\mathrm{b}}$ \\
\hline & & $\mathrm{RA}+1-\mathrm{MCP}$ & 0.0 & \pm & $0.0^{\mathrm{a}}$ & 2.9 & \pm & $2.9^{\mathrm{a}}$ & 0.0 & \pm & $0.0^{\mathrm{a}}$ & 12.4 & \pm & $5.9^{\mathrm{a}}$ \\
\hline \multirow{6}{*}{$\begin{array}{l}\text { Vascular } \\
\text { browning (\%) }\end{array}$} & \multirow{4}{*}{ I } & RA & 0.0 & \pm & $0.0^{\mathrm{a}}$ & 1.0 & \pm & $1.7^{\mathrm{a}}$ & 1.0 & \pm & $1.7^{\mathrm{a}}$ & 16.2 & \pm & $6.6^{\mathrm{b}}$ \\
\hline & & CA & 0.0 & \pm & $0.0^{\mathrm{a}}$ & 0.0 & \pm & $0.0^{\mathrm{a}}$ & 0.0 & \pm & $0.0^{\mathrm{a}}$ & 3.8 & \pm & $1.7^{\mathrm{a}}$ \\
\hline & & $\mathrm{RA}+1-\mathrm{MCP}$ & 0.0 & \pm & $0.0^{\mathrm{a}}$ & 3.8 & \pm & $6.6^{\mathrm{a}}$ & 2.9 & \pm & $2.9^{\mathrm{a}}$ & 10.5 & \pm & $3.3^{\mathrm{ab}}$ \\
\hline & & RA & 5.7 & \pm & $9.9^{\mathrm{a}}$ & 21.9 & \pm & $13.5^{\mathrm{b}}$ & 17.1 & \pm & $7.6^{\mathrm{b}}$ & 48.6 & \pm & $12.5^{\mathrm{b}}$ \\
\hline & \multirow[t]{2}{*}{ II } & CA & 0.0 & \pm & $0.0^{\mathrm{a}}$ & 9.5 & \pm & $3.3^{\mathrm{a}}$ & 2.9 & \pm & $0.0^{\mathrm{a}}$ & 11.4 & \pm & $5.7^{\mathrm{a}}$ \\
\hline & & $\mathrm{RA}+1-\mathrm{MCP}$ & 3.8 & \pm & $1.7^{\mathrm{a}}$ & 21.0 & \pm & $6.0^{\mathrm{b}}$ & 3.8 & \pm & $3.3^{\mathrm{a}}$ & 17.1 & \pm & $7.6^{\mathrm{a}}$ \\
\hline Interaction & & & & $\begin{array}{c}\text { External } \\
\text { damage }(\%)\end{array}$ & & & & & & $\begin{array}{c}\text { Gray } \\
\text { pulp (\%) }\end{array}$ & & & $\begin{array}{c}\text { Vascular } \\
\text { browning } \\
(\%)\end{array}$ & \\
\hline Treatment (A) & & & & $0.0056^{*}$ & & & & & & $<0.0001^{*}$ & & & $<0.0001^{*}$ & \\
\hline Storage time (B) & & & & $<0.0001^{*}$ & & & & & & $<0.0001^{*}$ & & & $<0.0001^{*}$ & \\
\hline Harvest (C) & & & & 0.535 & & & & & & $0.0004^{*}$ & & & $<0.0001^{*}$ & \\
\hline$A \times B$ & & & & 0.3752 & & & & & & $0.0001^{*}$ & & & $0.0323^{*}$ & \\
\hline $\mathrm{A} \times \mathrm{C}$ & & & & $<0.0001^{*}$ & & & & & & 0.0922 & & & $0.0114^{*}$ & \\
\hline $\mathrm{B} \times \mathrm{C}$ & & & & $0.0055^{*}$ & & & & & & $0.0031^{*}$ & & & $0.0053^{*}$ & \\
\hline $\mathrm{A} \times \mathrm{B} \times \mathrm{C}$ & & & & 0.153 & & & & & & $0.0064^{*}$ & & & 0.2605 & \\
\hline
\end{tabular}

Factor: Treatment: RA, CA, and RA + 1-MCP. Storage time: fruits storage for 30 and 50 days at $5^{\circ} \mathrm{C}$. Harvest: two harvest, early, and advanced maturity stages. Sample significant.

increase with exposure to the air [34]. In fruit stored for $30 \mathrm{~d}$ in RA, independent of the maturity stage, an incidence of pulp spot close to $30 \%$ was observed after $50 \mathrm{~d}$ of storage (Figure $3(\mathrm{a})$ ). The severity of this disorder observed after $30 \mathrm{~d}$ or $50 \mathrm{~d}$ of storage in RA was mainly moderate to severe (Figure 3(b)). Fruits at the advanced maturity stage stored under CA for $50 \mathrm{~d}$ showed a low incidence of this disorder, approximately 5\% (Figure 3(a)). Fruit at the advanced maturity stage treated with 1 -MCP had a $30 \%$ incidence of pulp spot after $30 \mathrm{~d}$ that decreased, approaching 10\%, after $50 \mathrm{~d}$ of storage (Figure 3(a)). This lower susceptibility to pulp spot would be explained by an improved capacity of these avocados to withstand low temperature at the advanced maturity stage, at least with respect to this disorder [37]. After a shelf life time at $20^{\circ} \mathrm{C}$ no major changes were observed both in the incidence and severity of pulp spot at both maturity stages (data not shown).

In the "Hass" variety, it has been shown that fruit harvested at the end of the season, with a higher oil content, usually shows a higher incidence of cold storage-induced disorders $[38,39]$, indicating that maturity at harvest and ripeness are important factors in determining avocado fruit susceptibility to physiological disorder development [33]. As observed for "Fuerte" and "Edranol" in this study, avocado harvested with a higher oil content (Harvest II) generally showed a higher incidence of gray pulp and vascular browning, independent of the oil content level at harvest in each variety, suggesting a senescence-related component due to fruit age on the tree. Interestingly, "Fuerte a variety with a higher oil content than "Edranol" showed higher levels of physiological disorders under RA storage. Internal browning disorders have been linked to peroxidase (POD) and polyphenol oxidase activities (PPO) [40]; however, other metabolic processes may be altered in cold-injured fruit [41]. For example, chilling injury-induced disorders are accompanied by alterations of cell membrane conformation and structure [42], particularly because of lipid degradation [43]. These metabolic 
TABLE 9: Evaluation of physiological disorders in "Edranol" avocado under different storage conditions for 30 and 50 days at $5^{\circ} \mathrm{C}$.

\begin{tabular}{|c|c|c|c|c|c|c|c|c|c|c|c|c|c|c|}
\hline \multirow{3}{*}{ Variable } & \multirow{3}{*}{ Harvest } & \multirow{3}{*}{ Treatment } & \multicolumn{12}{|c|}{ Storage time (days) } \\
\hline & & & \multicolumn{3}{|c|}{$30 \mathrm{~d}$} & \multicolumn{3}{|c|}{$30+\mathrm{SL}$} & \multicolumn{3}{|c|}{$50 \mathrm{~d}$} & \multicolumn{3}{|c|}{$50+\mathrm{SL}$} \\
\hline & & & Mean & & $\mathrm{SD}$ & Mean & & $\mathrm{SD}$ & Mean & & SD & Mean & & SD \\
\hline \multirow{6}{*}{$\begin{array}{l}\text { External } \\
\text { damage (\%) }\end{array}$} & \multirow{4}{*}{ I } & RA & 77.1 & \pm & $14.3^{\mathrm{b}}$ & 94.3 & \pm & $0.0^{\mathrm{b}}$ & 92.4 & \pm & $1.7^{\mathrm{c}}$ & 100.0 & \pm & $0.0^{\mathrm{c}}$ \\
\hline & & $\mathrm{CA}$ & 0.0 & \pm & $0.0^{\mathrm{a}}$ & 0.0 & \pm & $0.0^{\mathrm{a}}$ & 1.0 & \pm & $1.7^{\mathrm{a}}$ & 2.9 & \pm & $2.9^{\mathrm{a}}$ \\
\hline & & $\mathrm{RA}+1-\mathrm{MCP}$ & 0.0 & \pm & $0.0^{\mathrm{a}}$ & 0.0 & \pm & $0.0^{\mathrm{a}}$ & 34.3 & \pm & $10.3^{\mathrm{b}}$ & 34.3 & \pm & $10.3^{\mathrm{b}}$ \\
\hline & & RA & 85.7 & \pm & $24.7^{\mathrm{b}}$ & 87.6 & \pm & $21.4^{\mathrm{b}}$ & 57.1 & \pm & $5.0^{\mathrm{c}}$ & 85.7 & \pm & $7.6^{\mathrm{c}}$ \\
\hline & \multirow[t]{2}{*}{ II } & $\mathrm{CA}$ & 0.0 & \pm & $0.0^{\mathrm{a}}$ & 1.0 & \pm & $1.7^{\mathrm{a}}$ & 1.0 & \pm & $1.7^{\mathrm{a}}$ & 1.0 & \pm & $1.7^{\mathrm{a}}$ \\
\hline & & $\mathrm{RA}+1-\mathrm{MCP}$ & 0.0 & \pm & $0.0^{\mathrm{a}}$ & 1.0 & \pm & $1.7^{\mathrm{a}}$ & 34.3 & \pm & $10.3^{\mathrm{b}}$ & 39.0 & \pm & $16.3^{b}$ \\
\hline \multirow{6}{*}{ Gray pulp (\%) } & \multirow{4}{*}{ I } & $\mathrm{RA}$ & 0.0 & \pm & $0.0^{\mathrm{a}}$ & 24.8 & \pm & $3.3^{\mathrm{b}}$ & 4.8 & \pm & $1.7^{\mathrm{b}}$ & 12.4 & \pm & $9.2^{\mathrm{b}}$ \\
\hline & & CA & 0.0 & \pm & $0.0^{\mathrm{a}}$ & 0.0 & \pm & $0.0^{\mathrm{a}}$ & 0.0 & \pm & $0.0^{\mathrm{a}}$ & 0.0 & \pm & $0.0^{\mathrm{a}}$ \\
\hline & & $\mathrm{RA}+1-\mathrm{MCP}$ & 0.0 & \pm & $0.0^{\mathrm{a}}$ & 1.0 & \pm & $1.7^{\mathrm{a}}$ & 0.0 & \pm & $0.0^{\mathrm{a}}$ & 0.0 & \pm & $0.0^{\mathrm{a}}$ \\
\hline & & RA & 1.0 & \pm & $1.7^{\mathrm{a}}$ & 3.8 & \pm & $4.4^{\mathrm{a}}$ & 20.0 & \pm & $15.1^{\mathrm{b}}$ & 56.2 & \pm & $22.3^{\mathrm{b}}$ \\
\hline & \multirow[t]{2}{*}{ II } & $\mathrm{CA}$ & 0.0 & \pm & $0.0^{\mathrm{a}}$ & 0.0 & \pm & $0.0^{\mathrm{a}}$ & 0.0 & \pm & $0.0^{\mathrm{a}}$ & 1.0 & \pm & $1.7^{\mathrm{a}}$ \\
\hline & & $\mathrm{RA}+1-\mathrm{MCP}$ & 0.0 & \pm & $0.0^{\mathrm{a}}$ & 0.0 & \pm & $0.0^{\mathrm{a}}$ & 0.0 & \pm & $0.0^{\mathrm{a}}$ & 1.0 & \pm & $1.7^{\mathrm{a}}$ \\
\hline \multirow{6}{*}{$\begin{array}{l}\text { Vascular } \\
\text { browning (\%) }\end{array}$} & \multirow{4}{*}{ I } & RA & 0.0 & \pm & $0.0^{\mathrm{a}}$ & 16.2 & \pm & $7.2^{\mathrm{a}}$ & 13.3 & \pm & $9.2^{\mathrm{b}}$ & 35.2 & \pm & $9.2^{\mathrm{b}}$ \\
\hline & & CA & 0.0 & \pm & $0.0^{\mathrm{a}}$ & 6.7 & \pm & $7.2^{\mathrm{a}}$ & 0.0 & \pm & $0.0^{\mathrm{a}}$ & 0.0 & \pm & $0.0^{\mathrm{a}}$ \\
\hline & & $\mathrm{RA}+1-\mathrm{MCP}$ & 0.0 & \pm & $0.0^{\mathrm{a}}$ & 3.8 & \pm & $6.6^{\mathrm{a}}$ & 0.0 & \pm & $0.0^{\mathrm{a}}$ & 0.0 & \pm & $0.0^{\mathrm{a}}$ \\
\hline & & RA & 0.0 & \pm & $0.0^{\mathrm{a}}$ & 6.7 & \pm & $6.0^{\mathrm{b}}$ & 11.4 & \pm & $12.5^{\mathrm{b}}$ & 41.9 & \pm & $15.0^{\mathrm{b}}$ \\
\hline & \multirow[t]{2}{*}{ II } & $\mathrm{CA}$ & 0.0 & \pm & $0.0^{\mathrm{a}}$ & 1.0 & \pm & $1.7^{\mathrm{a}}$ & 1.0 & \pm & $1.7^{\mathrm{a}}$ & 2.9 & \pm & $2.9^{\mathrm{a}}$ \\
\hline & & $\mathrm{RA}+1-\mathrm{MCP}$ & 0.0 & \pm & $0.0^{\mathrm{a}}$ & 0.0 & \pm & $0.0^{\mathrm{a}}$ & 0.0 & \pm & $0.0^{\mathrm{a}}$ & 1.0 & \pm & $1.7^{\mathrm{a}}$ \\
\hline Interaction & & & & $\begin{array}{c}\text { External } \\
\text { damage } \\
(\%)\end{array}$ & & & & & & $\begin{array}{c}\text { Gray } \\
\text { pulp (\%) }\end{array}$ & & & $\begin{array}{c}\text { Vascular } \\
\text { browning } \\
(\%)\end{array}$ & \\
\hline Treatment (A) & & & & $<0.0001^{*}$ & & & & & & $<0.0001^{*}$ & & & $<0.0001^{*}$ & \\
\hline $\begin{array}{l}\text { Storage } \\
\text { time (B) }\end{array}$ & & & & $<0.0001^{*}$ & & & & & & $<0.0001^{*}$ & & & $<0.0001^{*}$ & \\
\hline Harvest (C) & & & & 0.2562 & & & & & & 0.0698 & & & 0.5361 & \\
\hline $\mathrm{A} \times \mathrm{B}$ & & & & $<0.0001^{*}$ & & & & & & $<0.0001^{*}$ & & & $<0.0001^{*}$ & \\
\hline $\mathrm{A} \times \mathrm{C}$ & & & & 0.1244 & & & & & & 0.1062 & & & 0.6971 & \\
\hline $\mathrm{B} \times \mathrm{C}$ & & & & $0.0342^{*}$ & & & & & & $<0.0001^{*}$ & & & $0.0107^{*}$ & \\
\hline $\mathrm{A} \times \mathrm{B} \times \mathrm{C}$ & & & & $0.0347^{*}$ & & & & & & $<0.0001^{*}$ & & & 0.9898 & \\
\hline
\end{tabular}

RA: regular air (85\% relative humidity); CA: controlled atmosphere $\left(4 \% \mathrm{O}_{2}+6 \% \mathrm{CO}_{2}\right)$; 1-MCP: 1-methylcyclopropene (300 ppb); s.d: standard deviation. I: early maturity stage; II: advanced maturity stage; d: days at $5{ }^{\circ} \mathrm{C}$. SL (shelf life): fruit storage at $20^{\circ} \mathrm{C}$. Fruit at SL was evaluated at the same flesh firmness level. Each value corresponds to mean of 35 fruits. Different letters within each storage time and harvest represent significant differences at $P \leq 0.05$ as determined by the LSD test. Factor: treatment: RA, CA, and RA + 1-MCP. Storage time: fruits storage for 30 and 50 days at $5^{\circ} \mathrm{C}$. Harvest: two harvest, early and advanced maturity stages. *Sample significant, $P<0.05$.

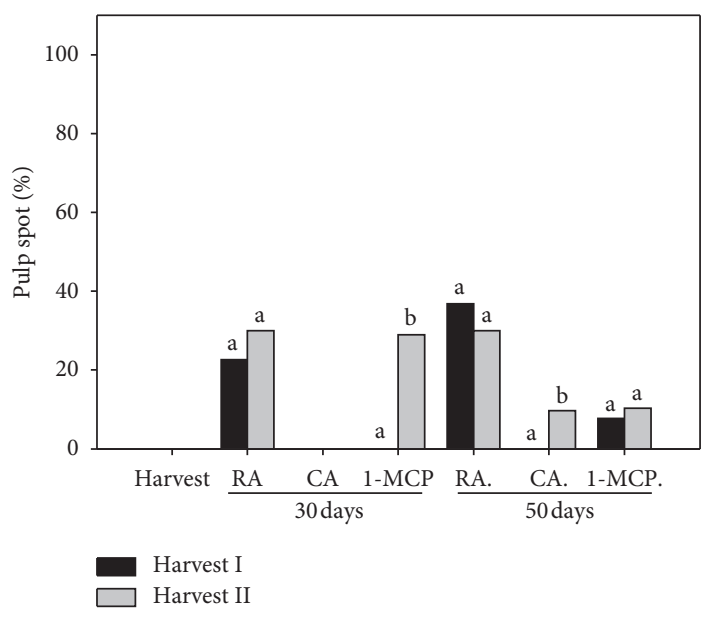

(a)

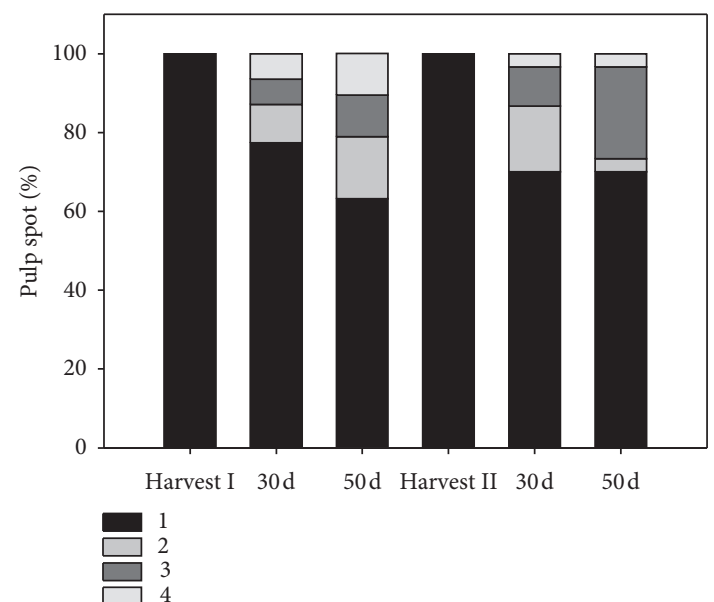

(b)

FIgURE 3: Continued. 


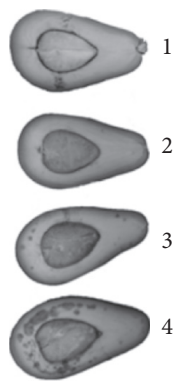

(c)

Figure 3: (a) Incidence of pulp spot in "Fuerte" avocado immediately after 30 and 50 days at $5^{\circ} \mathrm{C}$. (b) Severity, expressed as percentage by category, in fruit stored after for 30 or 50 days at $5^{\circ} \mathrm{C}$ under RA. (c) Hedonic scale (1-4): 1, no occurrence; 2, slight; 3, moderate; 4, severe. Different letters within each storage period and treatment represent significant differences at $P \leq 0.05$ as determined by the LSD test. d: days.

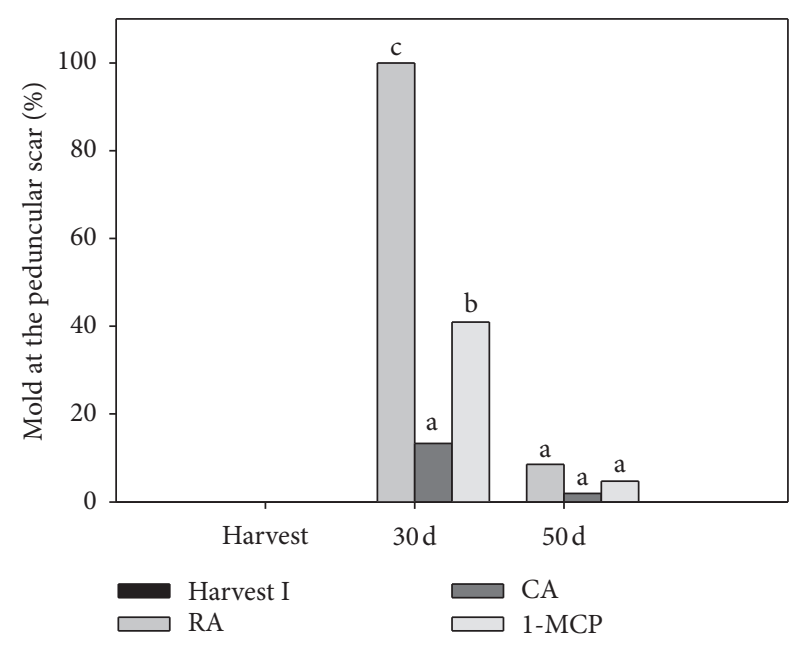

(a)

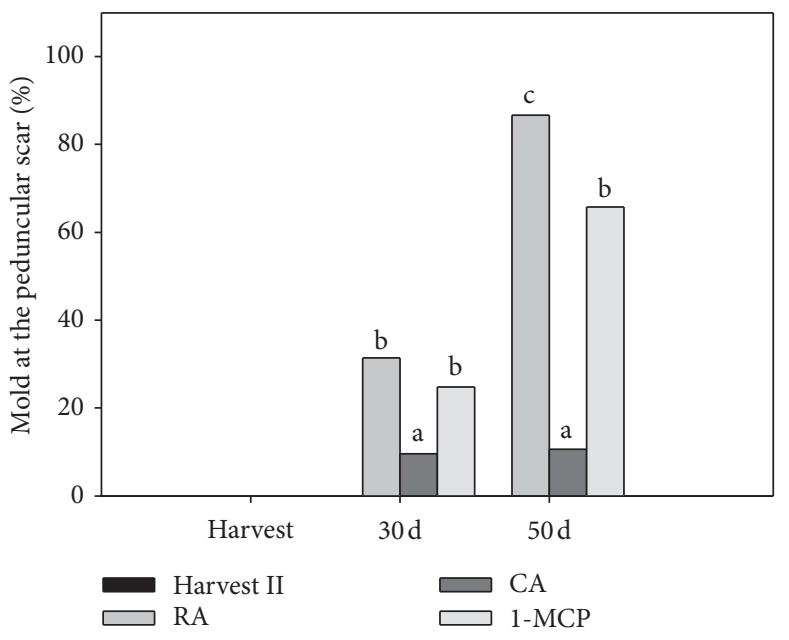

(b)

FIgURE 4: Incidence (\%) of mold at the peduncular scar immediately after storage in "Edranol" avocado. (a) Harvest I and (b) Harvest II. RA: regular air. CA: controlled atmospheres $\left(4 \% \mathrm{O}_{2}\right.$ and $\left.6 \% \mathrm{CO}_{2}\right)$. Different letters within each storage period represent significant differences at $P \leq 0.05$ as determined by the LSD test. d: days.

processes trigger changes at the transcriptional and posttranscriptional levels and differential gene expression [42].

CA and 1-MCP have been extensively described as two technological approaches for reducing the incidence of physiological disorders in avocado. In this study, a CA storage using $4 \% \mathrm{CO}_{2}$ and $6 \% \mathrm{O}_{2}$ could delay and reduce the expression of disorders in the skin and flesh, was more effective after $30 \mathrm{~d}$ of storage, and maintained its benefits during shelf life for some disorders. Reduced $\mathrm{O}_{2}$ and increased $\mathrm{CO}_{2}$ had a major impact on ethylene metabolism, considering production and transcript expression levels. Similarly, the effectiveness of 1-MCP has been demonstrated in delaying the onset of ripening, resulting in a reduction in ethylene rate, respiration rate, softening rate, and physiological disorders, all features observed in "Fuerte" and "Edranol" in our study. These benefits would be achieved by affecting PPO and POD activities, the main enzymes involved in mesocarp discoloration in avocado varieties [26].

3.7. Mold Incidence. The incidence of mold at the peduncular scar of the fruit was mainly observed in "Edranol." In
"Fuerte," the mold incidence was much lower after both storage periods and did not show differences under the treatments evaluated (data not shown). Mold incidence was observed mainly in avocados stored under RA after $30 \mathrm{~d}$ (Figure 4(a)). After $50 \mathrm{~d}$ of storage, the incidence of this damage was observed in only $10 \%$ of the fruits evaluated (Figure 4(a)). The same phenomenon but with lower values was observed in fruit stored under CA and treated with 1MCP (Figure 4(a)). In fruit at the advanced maturity stage, the incidence of damage increased with the storage period, reaching $80 \%$ in avocados under RA after $50 \mathrm{~d}$ and $60 \%$ in 1 MCP-treated fruit. After $50 \mathrm{~d}$ of storage, fruits under CA showed only a $10 \%$ incidence of fungal damage, and no change was associated with the storage time (Figure 4(b)). After a shelf life time at $20^{\circ} \mathrm{C}$, no major changes were observed in the incidence of mold at both maturity stages (data not shown).

Ethylene inhibition by 1-MCP usually reduces the incidence of postharvest decay in climacteric fruit, including avocado [44]. However, the relationship between ethylene and fungus in postharvest fruits is still under study; whether the effect of this plant hormone occurs mainly through 
affecting fruit metabolism or by directly affecting the pathogens remains unclear [45]. It seems that during storage at $5^{\circ} \mathrm{C}, 1-\mathrm{MCP}$-treated avocados would show features similar to those of nonclimacteric fruit; for example, ethylene levels did not delay fungus growth during fruit ripening, which could explain the results observed in "Fuerte" in our study. 1-MCP has also been shown to prevent the accumulation of $(\mathrm{Z}, \mathrm{Z})-1$ acetoxy-2-hydroxy-4-oxo-heneicosa-12,15-diene (AFD), the most active antifungal compound in avocado, and this effect could also explain the higher incidence of mold observed in 1MCP-treated fruit [46]. However, further studies must be performed considering fungal species, fruit source, and environmental conditions including ethylene levels.

\section{Conclusion}

CA storage was shown to be very effective in delaying ripening and maintaining quality compared with RA storage. The application of 1-MCP was equally effective in "Edranol" and "Fuerte" avocados to delay softening, to delay ethylene and $\mathrm{CO}_{2}$ production during cold storage, and to downregulate the expression of genes involved in ethylene biosynthesis. In general, CA was most effective than 1-MCP in reducing most of the external and internal damages evaluated, and the effectiveness within each disorder was affected by maturity stage at harvest and evaluation time.

\section{Data Availability}

The data used to support the findings of this study are available from the corresponding author upon request.

\section{Conflicts of Interest}

The authors declare no conflicts of interest.

\section{Acknowledgments}

This work was fully funded by FONDECYT (CONICYT) under grant 1170501 .

\section{References}

[1] C. Vergara-Pulgar, K. Rothkegel, M. González-Agüero et al., "De novo assembly of Persea americana cv. "Hass" transcriptome during fruit development," BMC Genomics, vol. 20, no. 1, p. 108, 2019.

[2] V. C. Russo, E. R. Daiuto, R. L. Vietes, and R. E. Smith, "Postharvest parameters of the "Fuerte" avocado when refrigerated in different modified atmospheres," Journal of Food Processing and Preservation, vol. 38, no. 4, pp. 2006-2013, 2014.

[3] S. A. Rivera, R. Ferreyra, P. Robledo et al., "Identification of preharvest factors determining postharvest ripening behaviors in "Hass" avocado under long term storage," Scientia Horticulturae, vol. 216, pp. 29-37, 2017.

[4] D. Ahmed, F. Ahmed, A. El-Mongy, B. Abu-Aziz, and A. Yousef, "Postharvest storage of Hass and Fuerte avocados under modified atmosphere conditions," Journal of Applied Sciences Research, vol. 3, no. 4, pp. 267-274, 2007.
[5] J. P. Bower, J. G. Cutting, and A. B. Truter, "Modified atmosphere storage and transport of avocados-what does it mean," South Africa Avocado Growers Association Yearbook, vol. 12, pp. 17-20, 1989.

[6] G. J. Eksteen and A. B. Truter, "Effects of controlled and modified atmosphere storage on quality of eating ripe avocados," South African Avocado Growers' Association Yearbook, vol. 8, pp. 78-80, 1985.

[7] D. Lemmer, J. Bezuidenhout, S. Sekhune et al., "Semi-commercial evaluation of Smartfresh Tm with South African export avocados in static containers at a packinghouse during 2002," in Proceedings of the V World Avocado, Málaga, Spain, October 2003.

[8] A. B. Woolf, C. Requejo-Tapia, K. A. Cox et al., "1-MCP reduces physiological storage disorders of "Hass" avocados," Postharvest Biology and Technology, vol. 35, no. 1, pp. 43-60, 2005.

[9] B. G. Defilippi, A. M. Dandekar, and A. A. Kader, "Relationship of ethylene biosynthesis to volatile production, related enzymes, and precursor availability in apple peel and flesh tissues," Journal of Agricultural and Food Chemistry, vol. 53, no. 8, pp. 3133-3141, 2005.

[10] J. Jeong, D. J. Huber, and S. A. Sargent, "Influence of 1methylcyclopropene (1-MCP) on ripening and cell-wall matrix polysaccharides of avocado (Persea americana) fruit," Postharvest Biology and Technology, vol. 25, no. 3, pp. 241-256, 2002.

[11] D. Lemmer, F. J. Kruger, T. R. Malumane, and K. Y. Nxudu, "1-methylcyclopropane (1-MCP): an alternative for controlled atmosphere storage of South African export avocados," South African Avocado Growers' Association Yearbook, vol. 25, pp. 25-34, 2002.

[12] J. Jeong and D. J. Huber, "Suppression of avocado (Persea americana mill.) fruit softening and changes in cell wall matrix polysaccharides and enzyme activities: differential responses to 1-MCP and delayed ethylene application," Journal of the American Society for Horticultural Science, vol. 129, no. 5, pp. 752-759, 2004.

[13] B. G. Defilippi, T. Ejsmentewicz, M. P. Covarrubias, O. Gudenschwager, and R. Campos-Vargas, "Changes in cell wall pectins and their relation to postharvest mesocarp softening of "Hass" avocados (Persea americana Mill.)," Plant Physiology and Biochemistry, vol. 128, pp. 142-151, 2018.

[14] O. Gudenschwager, M. García-Rojas, B. G. Defilippi, and M. González-Agüero, "Identification and characterization of two putative genes encoding acetyl-coenzyme a carboxylase subunits that are possibly associated with internal browning during cold storage of "Hass" avocados (Persea americana Mill.)," Postharvest Biology and Technology, vol. 84, pp. 74-80, 2013.

[15] O. Gudenschwager, M. González-Agüero, and B. G. Defilippi, "A general method for high-quality RNA isolation from metabolite-rich fruits," South African Journal of Botany, vol. 83, pp. 186-192, 2012.

[16] V. Hershkovitz, H. Friedman, E. Goldschmidt, O. Feygenberg, and E. Pesis, "Induction of ethylene in avocado fruit in response to chilling stress on tree," Journal of Plant Physiology, vol. 166, no. 17, 2009.

[17] V. Hershkovitz, H. Friedman, E. Goldschmidt, and E. Pesis, "Ethylene regulation of avocado ripening differs between seeded and seedless fruit," Postharvest Biology and Technology, vol. 56, no. 2, pp. 138-146, 2010.

[18] M. García-Rojas, A. Morgan, O. Gudenschwager et al., "Biosynthesis of fatty acids-derived volatiles in "Hass" avocado is 
modulated by ethylene and storage conditions during ripening," Scientia Horticulturae, vol. 202, pp. 91-98, 2016.

[19] M. W. Pfaffl, "A new mathematical model for relative quantification in real-time RT-PCR," Nucleic Acids Research, vol. 29 , no. 9, p. 45, 2001.

[20] J. Jeong, D. J. Huber, and S. A. Sargent, "Delay of avocado (Persea americana) fruit ripening by 1-methylcyclopropene and wax treatments," Postharvest Biology and Technology, vol. 28, no. 2, 2003.

[21] E. Pesis, V. Krilo, O. Feygenberg, M. Ackerman, R. Ben-Arie, and D. Prusky, "Postharvest effect of 1-MCP on ripening of avocado cv. Ettinger," in Proceedings of the NATO Advanced Research Workshop, Biology and Biotechnology of the Plant Hormone Ethylene, Murcia, Spain, 2003.

[22] S. F. Yang and N. E. Hoffman, "Ethylene biosynthesis and its regulation in higher plants," Annual Review of Plant Physiology, vol. 35, no. 1, pp. 155-189, 1984.

[23] H. Kende, "Ethylene biosynthesis," Annual Review of Plant Physiology and Plant Molecular Biology, vol. 44, no. 1, pp. 283-307, 1993.

[24] C. E. Lewis, "The maturity of avocados-a general review," Journal of the Science of Food and Agriculture, vol. 29, no. 10, pp. 857-866, 1978.

[25] D. F. Faubion, F. G. Mitchell, G. Mayer, and M. L. Arpaia, "Response of "Hass" avocado fruit to postharvest storage in controlled atmosphere conditions," in Proceedings of the 2nd World Avocado Congress, vol. 1111, pp. 461-472, Orange, CA, USA, April 1992.

[26] V. Hershkovitz, S. I. Saguy, and E. Pesis, "Postharvest application of 1-MCP to improve the quality of various avocado cultivars," Postharvest Biology and Technology, vol. 37, no. 3, pp. 252-264, 2005

[27] M. I. Chitarra and A. B. Chitarra, "Pós-colheita de frutos e hortaliças: fisiología e manuseio," UFLA, vol. 785, 2005.

[28] S. Ben-Yehoshua and V. Rodov, "Transpiration and water stress, Postharvest physiology and pathology of vegetables," in Postharvest Physiology and Pathology of Vegetables, J. A. Bartz and J. K. Brecht, Eds., pp. 111-159, Marcel Dekker, New York, NY, USA, 2003.

[29] C. Espinosa-Cruz, S. Valle-Guadarrama, M. C. YbarraMoncada, and M. T. Martínez-Damián, "Comportamiento postcosecha de frutos de aguacate "Hass" afectado por temperatura y atmósfera modificada con microperforado," Revista Fitotecnia Mexicana, vol. 37, no. 3, pp. 235-242, 2014.

[30] A. B. Woolf, K. A. Cox, A. White, and I. B. Ferguson, "Low temperature conditioning treatments reduce external chilling injury of "Hass" avocados," Postharvest Biology and Technology, vol. 28, no. 1, pp. 113-122, 2003.

[31] P. Florissen, J. S. Ekman, C. Blumenthal, W. B. McGlasson, J. Conroy, and P. Holford, "The effects of short heat-treatments on the induction of chilling injury in avocado fruit (Persea americana Mill.)," Postharvest Biology and Technology, vol. 8, no. 2, pp. 129-141, 1996.

[32] J. Covarrubias, L. Lizana, and L. Luchsinger, "Incidence of cold storage of Esther avocado (Persea americana mill.) fruit on the occurrence of postharvest physiological disorders," in Proceedings of the VI World Avocado Congress, Viña del Mar, Chile, November 2007.

[33] M. García-Rojas, O. Gudenschwager, B. G. Defilippi, and M. González-Agüero, "Identification of genes possibly related to loss of quality in late-season "Hass" avocados in Chile," Postharvest Biology and Technology, vol. 73, pp. 1-7, 2012.
[34] A. White, A. B. Woolf, P. J. Hofman, and M. L. Arpaia, The International Avocado Quality Manual, University of California, Davis, CA, USA, 2009.

[35] E. Pesis, M. Ackerman, R. Ben-Arie et al., "Ethylene involvement in chilling injury symptoms of avocado during cold storage," Postharvest Biology and Technology, vol. 24, no. 2, pp. 171-181, 2002.

[36] S. F. Plessis and T. J. Koen, "Relationship between mineral nutrition and postharvest fruit disorders of "Fuerte" avocados," in Proceedings of the Second World Avocado Congress, pp. 395-402, Viña del Mar, Chile, 1994.

[37] P. Smith and R. Lunt, "Natalie robinson cole: the American Clzek?" Art Education, vol. 37, no. 1, pp. 36-37, 1984.

[38] J. Dixon, New Zealand Avocado Fruit Assessment Manual, Version 3.0, New Zealand Avocado Industry Council Ltd., Tauranga, New Zealand, 2003.

[39] J. Dixon, H. A. Pak, A. J. Mandemaker, D. B. Smith, T. A. Elmsly, and J. G. Cutting, "Fruit age management: the key to successful long distance export of New Zealand avocados, New Zealand avocado Growers' association," Annual Research Report, vol. 3, pp. 60-65, 2003.

[40] T. Wongsheree, S. Ketsa, and W. G. van Doorn, "The relationship between chilling injury and membrane damage in lemon basil (Ocimum $\times$ citriodourum) leaves," Postharvest Biology and Technology, vol. 51, no. 1, pp. 91-96, 2009.

[41] R. Nilo, C. Saffie, K. Lilley et al., "Proteomic analysis of peach fruit mesocarp softening and chilling injury using difference gel electrophoresis (DIGE)," BMC Genomics, vol. 11, no. 1, p. 43, 2010.

[42] C. Badea and K. S. Basu, "The effect of low temperature on metabolism of membrane lipids in plant and associated gene expression," Plant Omics, vol. 2, pp. 78-84, 2009.

[43] P. S. Campos, V. n. Quartin, J. c. Ramalho, and M. A. Nunes, "Electrolyte leakage and lipid degradation account for cold sensitivity in leaves of Coffea sp. plants," Journal of Plant Physiology, vol. 160, no. 3, pp. 283-292, 2003.

[44] C. H. Daulagala and W. A. M. Daundasekera, "Effect of 1methylcyclopropene (1-mcp) treatment on postharvest quality and antifungal activity of avocado cv. "pollock" under tropical storage conditions," Ceylon Journal of Science (Biological Sciences), vol. 44, no. 2, p. 75, 2016.

[45] P. Pristijono, R. Wills, L. Tesoriero, and J. Golding, "Ethylene on mycelial growth of postharvest fruit fungal pathogens," Horticulturae, vol. 4, no. 20, pp. 1-7, 2018.

[46] X. Wang, I. Kobiler, A. Lichter, A. Leikin-Frenkel, E. Pesis, and D. Prusky, "1-MCP prevents ethylene-induced accumulation of antifungal diene in avocado fruit," Physiological and Molecular Plant Pathology, vol. 67, no. 3-5, pp. 261-267, 2006. 